\title{
Keck Planet Imager and Characterizer: a dedicated single-mode fiber injection unit for high-resolution exoplanet spectroscopy
}

\author{
Jacques-Robert Delorme $\odot$, a,b, $*$ Nemanja Jovanovic $\odot,{ }^{a}$ Daniel Echeverri $\odot,{ }^{\mathbf{a}}$ \\ Dimitri Mawet, ${ }^{\text {a,c }}$ J. Kent Wallace $\odot,{ }^{\text {c }}$ Randall D. Bartos, ${ }^{c}$ Sylvain Cetre, ${ }^{b}$ \\ Peter Wizinowich $\odot$, Sam Ragland $\odot{ }^{\text {b }}$ Scott Lilley ${ }^{b},{ }^{b}$ Edward Wetherell, ${ }^{b}$

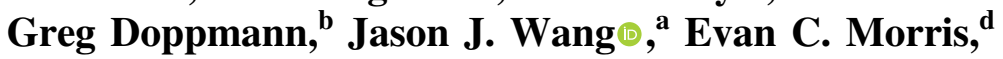 \\ Jean-Baptiste Ruffio $\odot,{ }^{a}$ Emily C. Martin $\odot,{ }^{d}$ Michael P. Fitzgerald $\odot,{ }^{e}$ \\ Garreth Ruane $\odot$, ${ }^{\mathrm{c}}$ Tobias Schofield, ${ }^{\mathrm{a}}$ Nick Suominen, ${ }^{\mathrm{c}}$ Benjamin Calvin, \\ Eric Wang, ${ }^{\mathrm{e}}$ Kenneth Magnone, ${ }^{\mathrm{e}}$ Christopher Johnson $\odot{ }^{\mathrm{e}}{ }^{\mathrm{Ji}}$ M. Sohn, ${ }^{\mathrm{e}}$ \\ Ronald A. López, ${ }^{e}$ Charlotte Z. Bond, ${ }^{b}$ Jacklyn Pezzato, ${ }^{a}$ Jorge L. Sayson $\odot$,

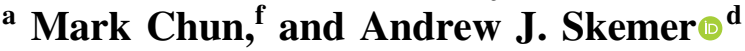 \\ ${ }^{a}$ California Institute of Technology, Department of Astronomy, Pasadena, California, \\ United States \\ ${ }^{b}$ W. M. Keck Observatory, Kamuela, Hawaii, United States \\ ${ }^{\mathrm{c} J e t}$ Propulsion Laboratory, California Institute of Technology, Pasadena, California, \\ United States \\ ${ }^{\mathrm{d}}$ University of California Santa Cruz, Santa Cruz, California, United States \\ e University of California, Department of Physics and Astronomy, Los Angeles, California, \\ United States \\ ${ }^{\mathrm{f}}$ University of Hawaii, Institute for Astronomy, Hilo, Hawaii, United States
}

\begin{abstract}
The Keck Planet Imager and Characterizer (KPIC) is a purpose-built instrument to demonstrate technological and instrumental concepts initially developed for the exoplanet direct imaging field. Located downstream of the current Keck II adaptive optic (AO) system, KPIC contains a fiber injection unit (FIU) capable of combining the high-contrast imaging capability of the AOs system with the high dispersion spectroscopy capability of the current Keck high resolution infrared spectrograph (NIRSPEC). Deployed at Keck in September 2018, this instrument has already been used to acquire high-resolution spectra $(R>30,000)$ of multiple targets of interest. In the near term, it will be used to spectrally characterize known directly imaged exoplanets and low-mass brown dwarf companions visible in the northern hemisphere with a spectral resolution high enough to enable spin and planetary radial velocity measurements as well as Doppler imaging of atmospheric weather phenomena. Here, we present the design of the FIU, the unique calibration procedures needed to operate a single-mode fiber instrument and the system performance. (C) The Authors. Published by SPIE under a Creative Commons Attribution 4.0 Unported License. Distribution or reproduction of this work in whole or in part requires full attribution of the original publication, including its DOI. [DOI: 10.1117/1.JATIS.7.3.035006]
\end{abstract}

Keywords: instrumentation; W. M. Keck observatory; exoplanets; high contrast imaging; high dispersion coronagraphy; high-resolution spectroscopy.

Paper 20188AS received Dec. 31, 2020; accepted for publication Jul. 27, 2021; published online Aug. 13, 2021.

\section{Introduction}

Since the first exoplanet detections almost three decades ago, ${ }^{1,2}$ thousands have been detected and confirmed using various indirect and direct observing strategies. Each technique provides access to specific planet populations and allows the observer to retrieve specific parameters of

*Address all correspondence to Jacques-Robert Delorme, jrdelorme@gmail.com 
the systems observed. Characterization of these planetary systems is critical to understanding their properties, formation, and evolution. Photometric and spectroscopic data are particularly valuable as they give access to multiple parameters (such as orbital parameters, spin, temperature, atmospheric composition, and cloud coverage). High dispersion spectroscopy (HDS) has quickly been recognized as a powerful way to characterize exoplanet atmospheres, and some strategies have been developed to spectrally characterize exoplanets detected using indirect methods. ${ }^{3,4}$ However, these measurements were challenging until more advanced observing strategies became available and used with stable infrared spectrographs, such as Keck/ NIRSPEC ${ }^{5}$ or VLT/CRIRES. ${ }^{6}$ The first molecular detections in exoplanet atmospheres allowed observers to constraint orbital parameters and retrieve new information such as wind flow and molecule mixing ratios. ${ }^{7}$

In parallel, the adaptive optic (AO) system and the infrared detectors of VLT/NACO ${ }^{8,9}$ have been used to image the first exoplanet in $2004^{10}$ followed in 2008 by the detection of the first three companions to HR $8799,{ }^{11}$ using the high contrast imaging (HCI) capabilities of both Keck and Gemini telescopes. These high HCI techniques have since been improved and many new observing strategies developed. Today most of the large telescopes have HCI capabilities, which enabled the detection of a few dozen companions. Photometric and spectroscopic data have slowly been collected on all the companions imaged using low to medium resolving power instruments $\left(R \sim 10\right.$ to 5000), such as Keck/OSIRIS ${ }^{12}$ Keck/Nirc2, LBT/LMIRcam, ${ }^{13}$ Palomar/P1640, ${ }^{14}$ VLT/Sphere, ${ }^{15}$ Gemini/GPI,${ }^{16}$ or Subaru/CHARIS. ${ }^{17}$

The next step in the field is to push to higher resolving power $(R>30,000)$ to collect spectral data where absorption and emission lines begin to be resolved using the high dispersion coronagraphy (HDC) technique. ${ }^{18-22}$ This technique optimally combines HCI and HDS and provides the ability to do species-by-species molecular characterization (e.g., water, carbon monoxide, methane), thermal (vertical) atmospheric structure, planetary spin measurements (length of day), and potentially Doppler imaging of atmospheric (clouds) and/or surface features (continents versus oceans). ${ }^{20}$ As none of the current facility instruments at large telescopes offers both HCI and HDS capabilities together, several projects recently commenced to combine the HCI capabilities with the HDS capabilities of various instruments at those observatories. Among them the fiber injection unit (FIU) part of the Keck Planet Imager and Characterizer (KPIC) project presented in this paper, the Rigorous Exoplanetary Atmosphere Characterization with High dispersion coronography instrument $(\mathrm{REACH})^{23,24}$ and High-Resolution Imaging and Spectroscopy of Exoplanets (HiRISE) ${ }^{25}$ The first two projects are both transitioning from commissioning to early science at the time of writing of this article and offer complimentary wavelength coverage across the near-IR (NIR) on Mauna Kea (REACH operates from Y-H and the KPIC-FIU operates in K and L bands), whereas HiRISE is still in the development stage.

In this paper, we present a detailed overview of the design and performance of the dedicated FIU for the Keck II telescope. In Sec. 2, we briefly introduce the KPIC demonstrator in which the FIU is contained and present its goals and the requirement that must be satisfied to reach them. In Sec. 3, we present details of the design of the FIU, a quick description of the key components of NIRSPEC, and our studies into the two key properties of the FIU: background and throughput. In Sec. 4, we describe the main calibration procedures performed to prepare the FIU for science observations. Section 5 presents the acquisition sequence used during the night to acquire science data and we present some example data acquired on one of our first test targets (HR 7672 $\mathrm{B}^{26}$ ). In Sec. 6, we demonstrate the sorts of information that can be extracted from HDC data. Finally, Sec. 7 rounds out the paper with some concluding thoughts.

\section{Keck Planet Imager and Characterizer}

The KPIC is a demonstrator deployed at the W. M. Keck Observatory. Located downstream of the Keck II AO system, ${ }^{27}$ the goal of this platform is to demonstrate multiple new concepts to image and characterize exoplanets on-sky. To reduce the complexity of such a project, the project is broken into phases. Phase I, deployed at the summit of Maunakea in fall 2018, contains an infrared pyramid wavefront sensor (PyWFS) ${ }^{28}$ and the modules and components critical for the demonstration of the FIU. Figure 1 shows several pictures of the KPIC optomechanics before

J. Astron. Telesc. Instrum. Syst. $\quad$ 035006-2 Jul-Sep 2021 • Vol. 7(3) 

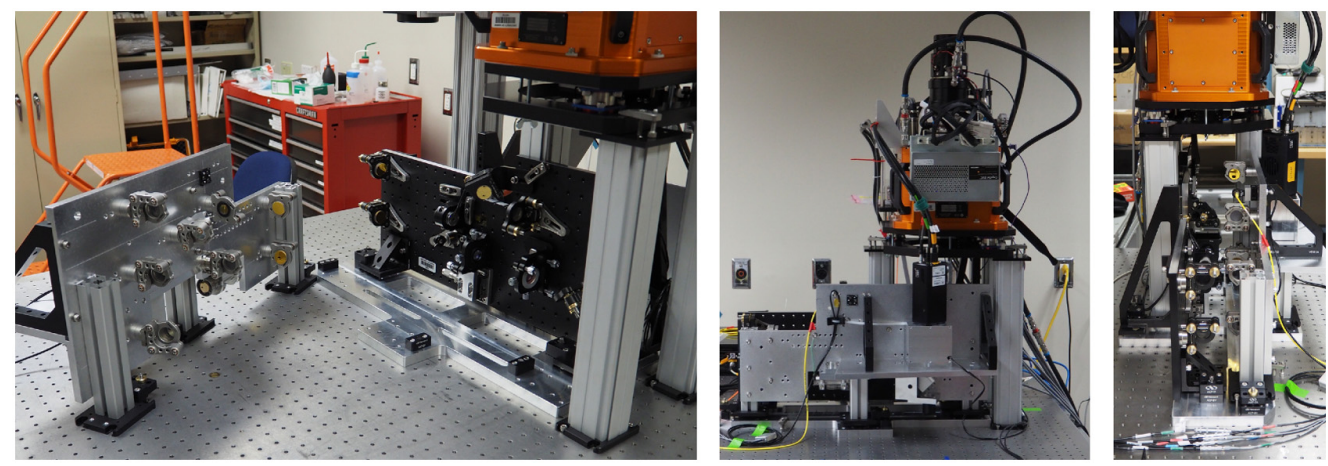

Fig. 1 Pictures of KPIC before its deployment.

their deployment. The vertical aluminum plate (unanodized) is the FIU plate. It supports optics common to both the FIU and PyWFS as well as the components for the FIU. The black anodized plate is the PyWFS plate. It supports all the optics of the PyWFS. The horizontal plate located under the two previous plates is a kinematic platform to simplify coalignment between the two vertical plates. The orange module supported by three struts is a SAPHIRA detector used by the PyWFS.

The second phase planned to deploy in late 2021 will contain extramodules: an atmospheric dispersion compensator (ADC), phase-induced amplitude apodization optics, a high order deformable mirror, and coronagraphs, whose goal will be to improve the overall throughput for the planet light while reducing star light leakage to reduce overall integration times. ${ }^{29-31}$ In this paper, we present a detailed overview of the first phase FIU and its performance.

\subsection{FIU Phase I Goals and Requirements}

The first version of the FIU presented in this paper has been designed as a prototype. The design has been driven by the space available in the Keck II AO bench, the existing science instruments available at Keck (i.e., NIRC2 and NIRSPEC) and the science goals. Its main goal was to demonstrate the concept by acquiring high-resolution spectral data on a variety of targets. We wanted the instrument to be able to observe all known substellar companions accessible to Keck, which sets most of the requirements as shown in Table 1.

To achieve this goal, the target or its host star must be observable by the Keck II AO system. Although the FIU can be used in combination with the visible Shack Hartmann wavefront sensor, we mainly use it with the IR PyWFS. Since its deployment, this wavefront sensor, which operates in $\mathrm{H}$ band, has been consistently used to observe targets up to 12th magnitude. The Strehl ratio measured in $\mathrm{K}$ band is $65 \%$ in 0.5 arc sec seeing and $45 \%$ in 1 arc sec seeing when the $\mathrm{H}$ magnitude of the star observed is $\leq 6$. Above this magnitude performance progressively decreases. The performance of this wavefront sensor has been described in detail in Ref. 28. It is currently not possible to close the AO loop on a system with two or more components if they have a similar $\mathrm{H}$ magnitude and a separation between 0.5 and 2 arc sec. This issue will be addressed when the second phase will be deployed. Because the PyWFS is using most of the $\mathrm{H}$ band light and the targets of interest are brighter in the $\mathrm{K}$ and $\mathrm{L}$ bands, the first version of the FIU was designed to collect spectral data in those photometric bands.

Once the AO loop is closed, the main challenge is to precisely align the object of interest with a single-mode fiber (SMF) with a precision of less than one-fifth of a $\lambda / D(\leq 10$ mas in $\mathrm{K}$ band) to achieve and maintain high coupling. To align the object of interest with an SMF, we image the system on a detector. Usually not visible on this detector, the object of interest is aligned with an SMF by tracking the position of its host star at a relatively low frequency $(<1 \mathrm{~Hz})$. This camera is sensitive to both $\mathrm{J}$ and $\mathrm{H}$ bands. However, we permanently installed an $\mathrm{H}$ band filter in front of it. In the second phase of this project, we will install it in a filter wheel with multiple filter options. To observe all known substellar companions accessible to Keck, we must be able to track the position of object with an H-mag up to 12 and have a capture range of $\pm 2.5 \operatorname{arc}$ sec. 
Table 1 FIU phase I requirements.

\begin{tabular}{lcc}
\hline \hline Specifications & Value & Notes \\
\hline Tracking and acquisition & $\sim \lambda / D$ & SMF optimally matched to PSF \\
Scale of optical fibers & \pm 2.5 arc sec & Covers most high contrast targets \\
Capture range & $<0.2 \lambda / D$ & - \\
Alignment accuracy & $<1 \mathrm{~Hz}$ & Tracking, not tip/tilt correction \\
Tracking speed & $<0.2 \lambda / D$ RMS & - \\
Tracking stability & $\mathrm{J}$ or H band & PyWFS limitation \\
Tracking bands & $>12$ in H-mag & \\
Limiting magnitude & $\mathrm{K}$ and L bands & Not simultaneously \\
Spectroscopy & $\geq 30,000$ & NIRSPEC limitation \\
Spectral range & $\geq 2$ pixels & At all wavelengths \\
Resolving power & $\geq 16 \mathrm{~K}$-mag & From top of atmosphere \\
Sampling & & S/N $\geq 3$ in CCF in $\approx 2 \mathrm{~h}$ \\
Overall performance & & \\
Total peak efficiency & & \\
Pt sources sensitivity & &
\end{tabular}

The use of SMFs makes the observation with such a system more challenging than observations with conventional AO-fed slit spectrographs, such as Keck/NIRSPEC or VLT/CRIRES. However, they provide multiple benefits. Specifically, the SMF has a small solid angle on sky minimizing thermal background leaking into the spectrograph, which is critical in $\mathrm{K}$ and $\mathrm{L}$ bands. They can also help filter out unwanted speckle (star light), ${ }^{32}$ and they make future wavefront control relatively simple in that only a few spatial frequencies need to be addressed, ${ }^{33}$ which allows the bandwidth to be increased with these algorithms. ${ }^{34}$ Finally, they allow the use of compact fiber-fed spectrographs that can be located of the telescope in a more stable environment. If fed by an SMF, the position and shape of the beam inside a fiber-fed spectrograph is agnostic of input conditions and greatly simplifies the spectral calibration by making the line spread function, a Gaussian profile in this case, which is extremely stable.

To reduce the cost and the time needed for this project, we designed the FIU as an interface to connect the AO bench to NIRSPEC. Because NIRSPEC was not designed as a fiber-fed spectrograph, it constrained the design in multiple ways (shape of the cold stop, injection outside of the cryostat, size, and shape of the slits available, etc.) described later in this paper. NIRSPEC sets the resolving power to 35,000 if we maintain a sampling of at least 2 pixels at all wavelengths across the bands observed (K or L band). The data collected are compared with models by computing the cross correlation function $(\mathrm{CCF})$. To be usable, we must be able to detect the spectral signature of the object of interest with a signal-to-noise ratio (SNR) greater than 3 after $2 \mathrm{~h}$ of integration. Table 1 summarizes all the baseline requirements for the KPIC FIU.

\section{Fiber Injection Unit Design}

The FIU deployed at Keck is based on three modules: an injection module, a bundle of fibers, and an extraction module. This section describes these modules, the NIRSPEC instrument used to acquire the data, and the expected performances of the overall instrument. The interaction 
between these components and the way we are using them to achieve our goals are described in the following sections of this paper.

\subsection{Injection Module}

Located downstream of the Keck II AO system, the goal of the injection module is to inject the light of faint companions (exoplanets, brown dwarf, etc.) into one of the SMFs of a bundle described in Sec. 3.2. Figure 2 shows a schematic of the KPIC optical layout. This diagram is not to scale and the orientation of the optics is not correct. In this paper, we only describe the optical layout of the FIU.

To direct the light to the FIU, the converging beam coming from the AO bench is reflected by the three optics constituting a field steering mirror module (FSM, see Fig. 2). The first optic of this FSM (A) is the KPIC pick off. It consists of an actuated dichroic and a static flat mirror installed on a translation stage. When neither optic is in the beam, all the light goes to the facility infrared imager NIRC2. If the dichroic is in the beam, $90 \%$ of the $\mathrm{J}$ and $\mathrm{H}$ band light go to KPIC while the rest goes to NIRC2. If the flat mirror is in the beam, all the light goes to KPIC.

After the FSM, the converging beam is directed to the FIU plate. This plate is visible in the images presented in Fig. 1 (unanodized) and supports all the components of the injection module. After going through two optical relays (D-J in Fig. 2), comprised of simple reflective optics in phase I, the light is collimated by a $168.31-\mathrm{mm}$ focal length off axis parabola (OAP, K) made of Zerodur and coated with protected gold ( $\mathrm{Nu}-\mathrm{Tek})$. The two optical relays are based on four identical OAPs to the one used for collimation (Nu-Tek-D, G, K, J) and two flat mirrors (Newport-E and I). All these optics are made of Zerodur and coated with protected gold.

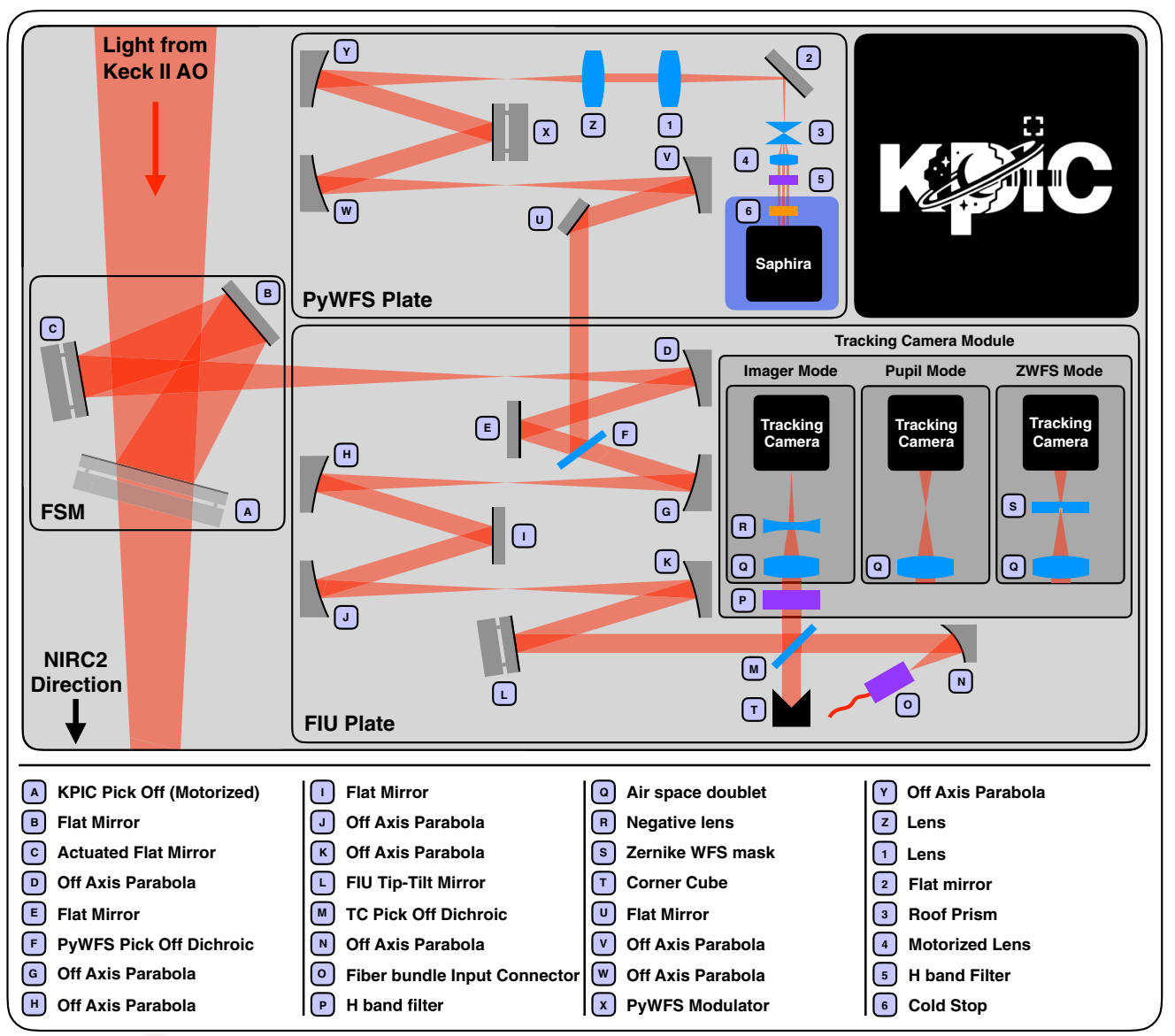

Fig. 2 Diagram of the KPIC optical layout. The scale and the orientation of the optics are not correct. The FIU and PyWFS plates as well as the Saphira detector are visible in the first figure of this paper. 
The $F \#$ of the input and output focal plane is the same as the $F \#$ of the telescope (13.66). The diameter of the collimated beams in these relays is $12.32 \mathrm{~mm}$. In the first optical relay, the static pick off dichroic of the PWS (Asahi Spectra-F) reflects $90 \%$ of the $\mathrm{J}$ and $\mathrm{H}$ band light in the direction of the sensor while the rest of the light is transmitted ( $\mathrm{K}$ and $\mathrm{L}$ band). These two optical relays that are mostly empty during the first phase of the project will be populated in phase two. ${ }^{30,31}$

After the two relays, a tip-tilt mirror (TTM) is situated in the pupil plane of the instrument for aligning the target with the fiber and fine tuning the pointing onto the fibers (L). The TTM (Physik Instrumente, S-330.8SL) is a piezo mechanism, which offers a field steering of $\pm 2.4 \mathrm{arc} \mathrm{sec}$ in two axes. An off-the-shelf-protected gold flat mirror (Thorlabs, $19 \mathrm{~mm}$ diameter) is attached to the platform using a custom-made, flexure-based mirror holder. Made of Invar and titanium, this mirror holder has been designed to minimize the forces applied to the flat mirror to minimize distortions and to be as light as possible to optimize the response of the overall TTM module.

A custom dichroic (Asahi Spectra, M) is used to split light and direct it to the camera for tracking purposes while allowing science light to pass through to the injection unit. It reflects $90 \%$ of the $\mathrm{J}$ and $\mathrm{H}$ band light while longer wavelength light ( $\geq \mathrm{K}$ and $\mathrm{L}$ ) is transmitted.

The reflected path toward the camera contains an $\mathrm{H}$ band filter (Asahi Spectra, P), an airspaced achromatic doublet (Thorlabs, Q), a planoconcave lens (Edmund Optics, R), and a lownoise InGaAs detector (First Light Imaging, Cred- $2^{35}$ ), which offers a $4.125 \times 5.125$ arc sec field-of-view (FOV, $512 \times 620$ pixels, with an $\approx 8.06$ mas/pixel sampling). When KPIC is used as an FIU, this detector is used to track the position of the science targets. For this reason, we refer to it as the "tracking camera." A stage can be used to translate the planoconcave lens in/out of the beam. When out of the beam, a pupil image of the Keck primary is formed. When in the beam, a focal plane image is formed. The FOV of this detector has been carefully selected to be able to observe the targets of interest selected for this project. It limits the maximum separation between the objects of the systems observed. However, this FOV allows us to observe most of the exoplanets already imaged and visible from Maunakea. The $\mathrm{K}$ and $\mathrm{L}$ band light is the next incident on the injection optic (N): a 35-mm focal length OAP (Nu-Tek). Made of Zerodur and coated with protected gold, this optic is the last of the FIU module before the fiber bundle (O) described in the following section. The bundle is carefully positioned at the focus $(F \#=2.8)$ and on the optical axis of the OAP. The effective focal length of the OAP is the one that maximizes the injection into the fiber for both $\mathrm{K}$ and $\mathrm{L}$ bands.

To aid in aligning the incoming beam with the bundle, a corner cube (T) is located beneath the dichroic. Its role will be explained in the calibration procedures of the system (see Sec. 4.1.1).

\subsection{Fiber Bundle}

The fiber bundle has several key roles: (1) it is used to route the science light to the spectrograph and (2) it has peripheral fibers that light can be reverse injected into to aid with alignment optimization. The layout of the bundle used in KPIC is shown in Fig. 3. On the input end, the fiber bundle is connected to the injection module (see Sec. 3.1) located inside the Keck AO bench. On the output side, the fiber bundle is connected to the extraction module (see Sec. 3.3) located in
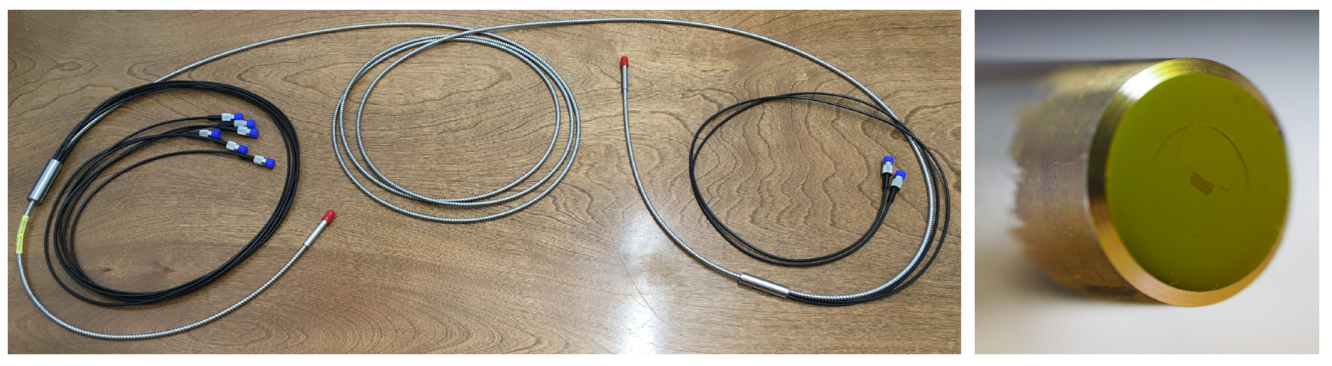

Fig. 3 Diagram the first KPIC-FIU fiber bundle. 
the calibration unit of NIRSPEC ${ }^{36}$ a facility class high spectral resolution spectrograph of the W. M. Keck Observatory.

Manufactured by Fiber Guide Industries, this critical component of the FIU is based on ZBLAN 6.5/125 fluoride fibers (Le Verre Fluoré) and SMF-28-ULTRA silica fibers (Corning). The bundle contains five ZBLAN 6.5/125 science fibers going from the input to the output connectors (note, this indicates the fibers have a $6.5-\mu \mathrm{m}$ diameter core and $125-\mu \mathrm{m}$ cladding). These are indicated in red on the diagram. This part of the bundle is $5 \mathrm{~m}$ long, a length necessary to connect the injection and extraction modules. The science fibers are surrounded by six SMF-28-ULTRA calibration fibers at the input connector end and two ZBLAN 6.5/125 calibration fibers on the output connector end, represented in blue and orange in the figure, respectively. These alignment fibers are contained within the furcation tubing of the main bundle for about $50 \mathrm{~cm}$ before they are broken out into individual fibers with FC/PC connectors. The input and output connectors also contain dummy fibers that act as scaffolding to locate the science and calibration fibers during manufacturing. Represented in black in the diagram, these fibers are segments of SMF-28-ULTRA terminated within the package.

The custom input and output connectors were manufactured using two different approaches compatible with the specific needs of each connector. The input connector contains three layers of fibers where the position of each fiber is not critical. In this connector, the fibers are butted cladding-to-cladding and their position is maintained by an outer metallic structure. The cladding-to-cladding construction maintains the core separation of two adjacent fibers to $\approx 125 \mu \mathrm{m}$, which corresponds to $\approx 800$ mas in $\mathrm{K}$ band.

The output connector contains only one layer of fibers but these fibers must be aligned with a high precision to make sure they pass through the mechanical slit of NIRSPEC downstream. For this reason, the fibers are supported using a V-groove on this side. The pitch of the V-groove used set the core-to-core separation between two adjacent fibers to $127 \mu \mathrm{m}$. On the input side, four of the input calibration fibers, labeled $C_{1}, C_{2}, C_{4}$, and $C_{6}$ in the diagram, are connected to a 1550$\mathrm{nm}$ laser source. The two other input calibration fibers $\left(C_{3}\right.$ and $\left.C_{5}\right)$ were connected to two high speed InGaAs photodetectors. On the output side, the two calibration fibers $\left(C_{A}\right.$ and $\left.C_{B}\right)$ were connected to two broadband infrared light sources (tungsten lamps, Thorlabs, SLS202L). In Sec. 4, we describe how and when these elements are used.

Figure 4 shows a picture of the fiber bundle currently used by KPIC [Fig. 4(a)] and a picture of the input connector [Fig. 4(b)]. For reference, the diameter of the connector is $7 \mathrm{~mm}$. The gold coating visible on the input connector is an antireflection (AR) coating to optimize the throughput of the bundle in $\mathrm{K}$ and $\mathrm{L}$ bands and is used at the output as well. The overall throughput of the bundle in $\mathrm{K}$ band was measured in the laboratory to be $95.3 \%$ in $\mathrm{K}$ band. According to the manufacturer, the attenuation of the ZBLAN fiber varied between 2.6 and $5.3 \mathrm{~dB} / \mathrm{km}$ across the K-band, which corresponds to a throughput $\geq 99.4 \%$ for $5 \mathrm{~m}$ of fiber. The rest of the throughput loss is due to the Fresnel reflections that occur at both ends of the bundle due to imperfect AR coatings. The L-band throughput has not been measured because of a lack of equipment in those bands. However, some preliminary observations in L-band indicate that the throughput of the bundle in this band is close to expectation.

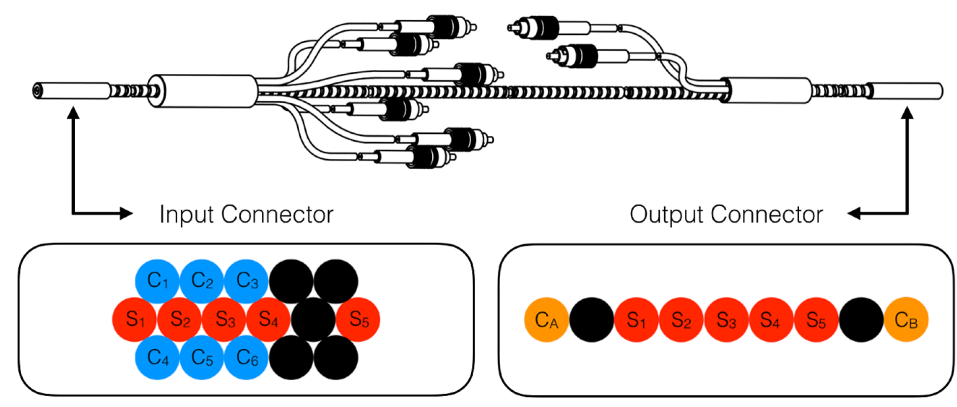

(a)

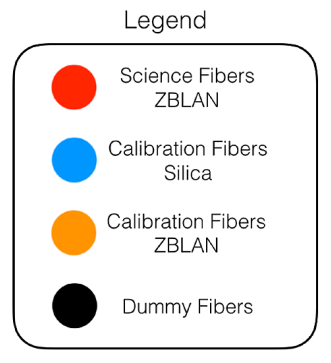

(b)

Fig. 4 (a) Picture of the bundle. (b) Picture of the input connector of the bundle. 
The minimum number of fibers needed to acquire HDC data is three: one for the target of interest, one for its host star, and one for the sky background. The final number of science fibers was chosen as a trade off between the price of the bundle and various technical constraints. For instance, the shape and size of the NIRSPEC slit limit the number of fibers on the output connector and impose a linear arrangement of fibers. We decided to use a design with five science fibers to cover most science cases and to have redundancy in case one of the science fibers was damaged. Regarding the calibration fibers, the bundle has been designed to be functional even if one of the calibration fibers of each set is damaged (one fiber connected to the laser, one to the photodetector, and one to the broadband light source). The position of the fibers on the input connector was not critical. We required the arrangement of fiber relatively to be compact to image most or all of them on the tracking camera if needed. To keep the system simple and efficient, we chose to maintain a coarse separation between the fibers. The minimum separation between two fibers is $\approx 800$ mas and the maximum is $\approx 4000$ mas. Because the star is usually brighter than the companion by several magnitudes, we can collect stellar light even if the star is not aligned with a fiber (light from the speckle field). We can adjust the amount of stellar light injected into a science fiber using the rotator of the telescope by bringing the star closer to a science fiber or moving it away. If the host star cannot be brought close enough to a science fiber when data are collected on the companion, we can collect spectral data on the host star before or/ and after by aligning it with one of the science fibers. Finally, because the separation between the first and last science fiber is $\approx 4$ arcsec, one of the fibers will always be far enough from the star to acquire background data.

\subsection{Extraction Module}

The extraction module, known as the fiber extraction unit (FEU), is the interface between the fiber bundle and NIRSPEC. Its goal is to reshape the light coming from the different fibers of the bundle and optimally inject this light into the high-resolution spectrograph, which was originally designed to work in the seeing limit. Figure 5(a) shows a picture of the FEU installed in the calibration unit of NIRSPEC, and Fig. 5(b) shows the computer-aided design (CAD) model.

A custom-made brass part visible in Fig. 5(b) (labeled 2) guides the output connector of the bundle (labeled 1) and aids in aligning it with an air-spaced doublet collimating lens mounted in the same part. This part has been made of brass mainly because this material is relatively soft and easy to machine. On the doublet side, we built a custom barrel to match the diameter of the lenses and to adjust the distance between the lenses if needed. For the bundle, we chose the material of the barrel to be softer than the material of the bundle (aluminum) to minimize the risk of galling when the bundle slides in the barrel. The doublet is composed of a germanium and silicon lens

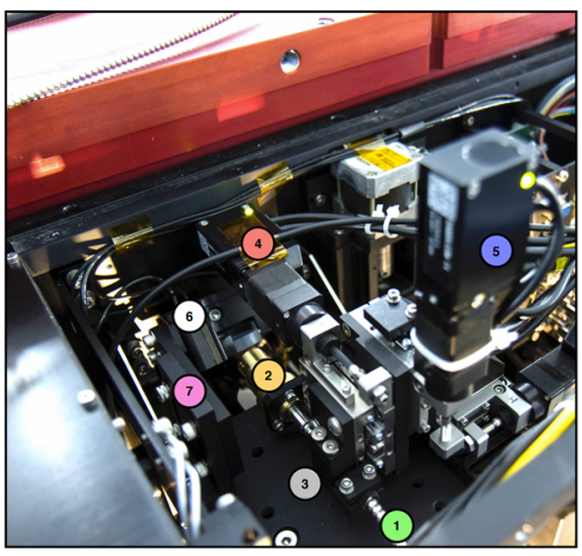

(a)

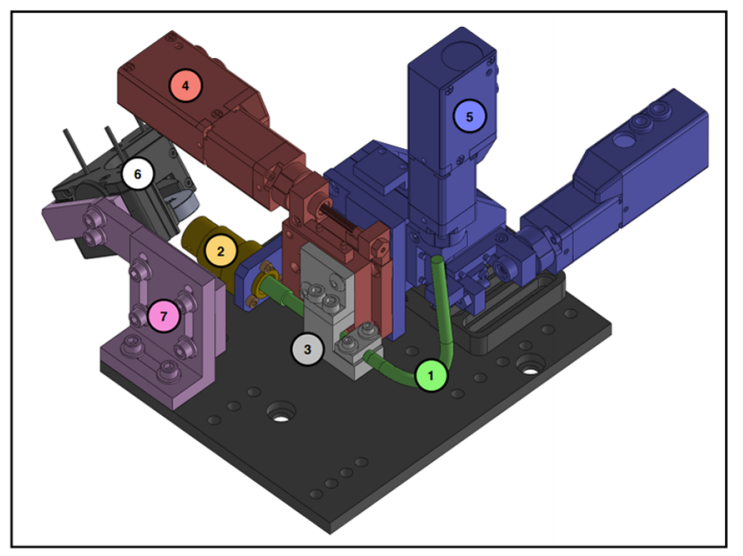

(b)

(1) Fiber Bundle (2) Lens/Bundle Holder (3) Bundle Kinematic (4) Focus Actuator (5) Field steering Act. (6) FEU TTM (7) TTM Bracket

Fig. 5 (a) Picture of the FEU installed in NIRSPEC's calibration unit. (b) A CAD model view of the FEU. 
(Rocky Mountain Instrument). The effective focal length of this doublet is $10.84 \mathrm{~mm}$, and the throughput in $\mathrm{K}$ and $\mathrm{L}$ bands was measured to be $>92 \%$. The numerical aperture of the science fiber has been measured in laboratory to be 0.175 at $2 \mu \mathrm{m}$. After the air-spaced doublet, the $1 / e^{2}$ diameter of the collimated Gaussian beam is $3.8 \mathrm{~mm}$. Close to its output connector, the stainless steel monocoil jacket of the bundle is attached to a motorized translation stage (labeled 4) using a custom-made kinematic interface (labeled 3). The translation stage is used to move the bundle along the optical axis guided by the brass part, which corresponds to the focus axis of the bundle/ lens system. The brass piece prevents the bundle from moving in the axes perpendicular to the optical axis and constrains the pitch and yaw of the output connector while the bundle is being focused. The rotation of the bundle about the optical axis is constrained by a clamp and kinematic interface. This simple kinematic interface combined with the brass holder proved reliable when it comes to returning the fiber bundle to the same position and orientation each time it has been connected. This assembly is mounted on two motorized translation stages, labeled 5, used as a pupil steering mechanism to move the fiber bundle and the air spaced doublet in the plane perpendicular to the optical axis. This mechanism is used each time the bundle is connected to align the beam coming from the bundle to the NIRSPEC field stop located into the cryostat. The next and last optical element of the FEU is a TTM (labeled 6) held by a custom-made bracket (labeled 7). Based on a Newport CONEX-AG-M100D, this element reflects the light in the direction of NIRSPEC calibration unit. This TTM is located in a pupil plane and is used to align the images of fibers with the slit of the spectrograph.

\subsection{NIRSPEC}

Figure 6 shows a diagram of the optical layout of NIRSPEC (not to scale). For more information regarding the NIRSPEC optical layout refer Refs. 36, 37, and 38. NIRSPEC's calibration unit located to the left side of this diagram contains the FEU described in the previous section.

A fold mirror (labeled D) installed in the calibration unit of NIRSPEC was motorized using a flip mechanism (OWIS, KSHM 65-LI-MDS). It is used to select the input of the calibration unit.

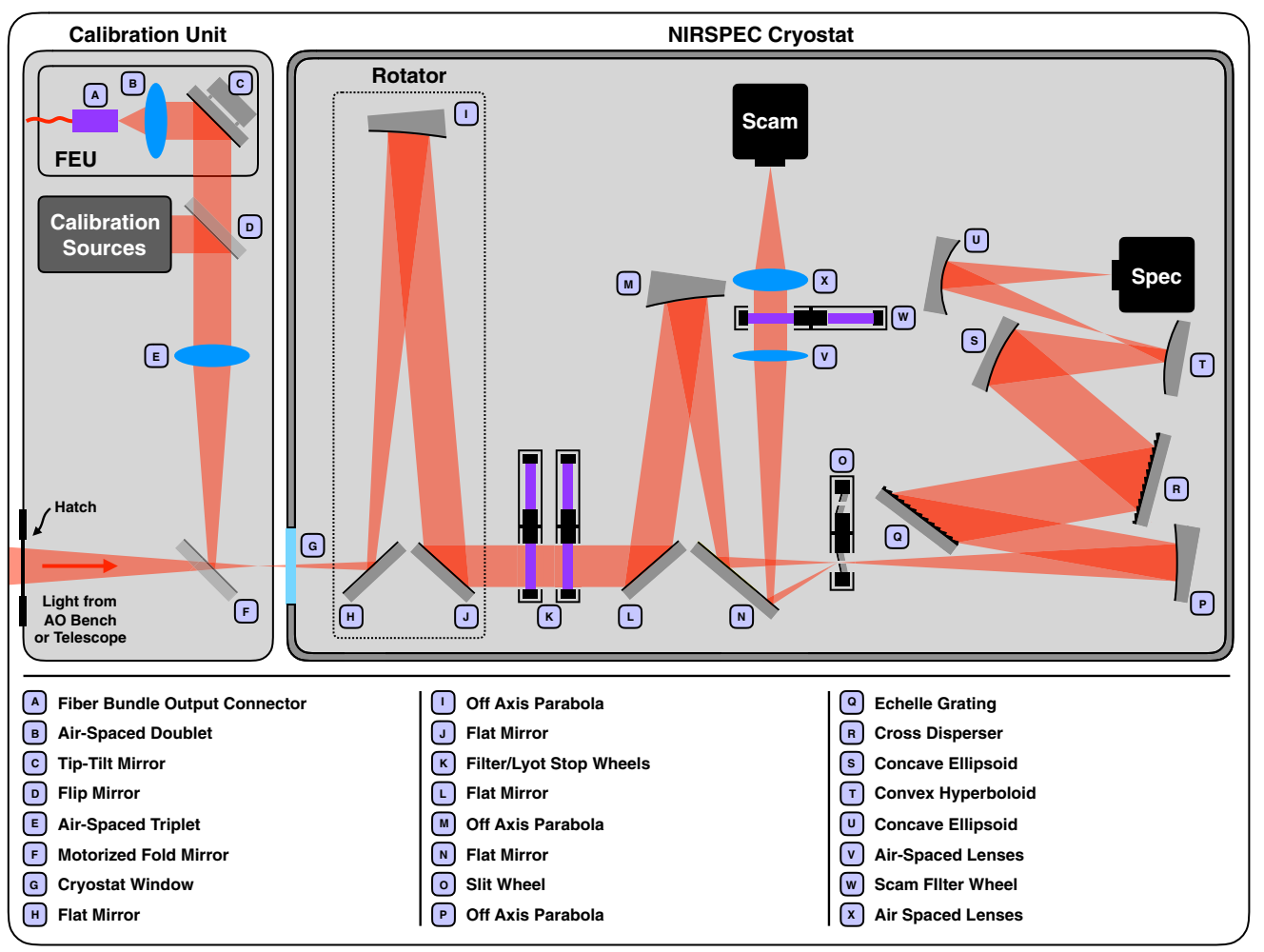

Fig. 6 Diagram of the NIRSPEC optical layout with the FEU installed in its calibration unit. This diagram is not to scale and does not show the optics in the correct orientation. 
When in the beam, the mirror directs light coming from the calibration sources of NIRSPEC's integrating sphere (and an air-spaced collimating triplet) to the slit to calibrate the spectrograph. When out of the beam, the light from the FEU is directed to NIRSPEC. After this optical element, the light is focused by an air-spaced triplet $(\mathrm{E}-\mathrm{EFL}=152.4 \mathrm{~mm})$ and reflected by a motorized fold mirror (F) before it enters into the NIRSPEC cryostat. When KPIC is in use, this fold mirror is in the beam. After this mirror, the beam goes through the uncoated $\mathrm{CaF}_{2}$ window of the NIRSPEC cryostat before being reflected by the three mirrors of the rotator. The second optic of this rotator $(\mathrm{I})$ is an OAP $(\mathrm{EFL}=400 \mathrm{~mm})$. After the rotator, the collimated Gaussian beam $\left(1 / e^{2}\right.$ diameter of $\left.10.0 \mathrm{~mm}\right)$ passes through two filter wheels $(\mathrm{K})$ before being focused by an OAP (M) onto the selected slit located in a slit wheel (O). All NIRSPEC slits are reflective. The light passing through the slit is dispersed and imaged on the science detector named "Spec" (Teledyne, science grade Hawaii-2RG detector). The light reflected by the slit is imaged by a slit-viewing camera named "Scam" (Teledyne, engineering grade Hawaii-2RG detector).

Figure 7 shows a combination of images acquired with the slit-viewing camera. This image has been obtained by combining images acquired with light injected into each fiber of the output connector of the bundle (see Sec. 3.2). This image presents the case where the PSFs associated with the fibers have been offset from the slit. This mode is mainly used during the calibration of the instrument. Indeed, unlike the science detector, the light coming from the fiber is not dispersed in the focal plane of the slit-viewing camera, which allows quick flux measurements. To align the PSFs and the slit, we use the FEU TTM (C). If the fibers contained in the bundle are not properly oriented with respect to the slit, we use the internal NIRSPEC rotator to make small adjustments.

Figure 8 shows two of the nine orders visible on the science detector when configured for $\mathrm{K}$ band observations. This image is also a combination of images obtained by combining images acquired with light injected into each fiber of the output connector of the bundle. Each order contains seven spectra. The five central spectra are associated with the five science fibers of the bundle, whereas the two lateral spectra are associated with the two calibration fibers. Depending on the slit selected (short or long), the light from the calibration fibers is reflected in the direction of Scam or transmitted to Spec. The separation between the spectra is fixed $(\approx 19$ pixels $)$ and set by the separation between the fibers in the output connector of the bundle. The width of the trace can be adjusted by moving the bundle along the optical axis using the focus actuator (labeled 4 in Fig. 5). We adjust the position of this actuator during a calibration procedure of the FEU (see Sec. 4.2) to properly sample the data.

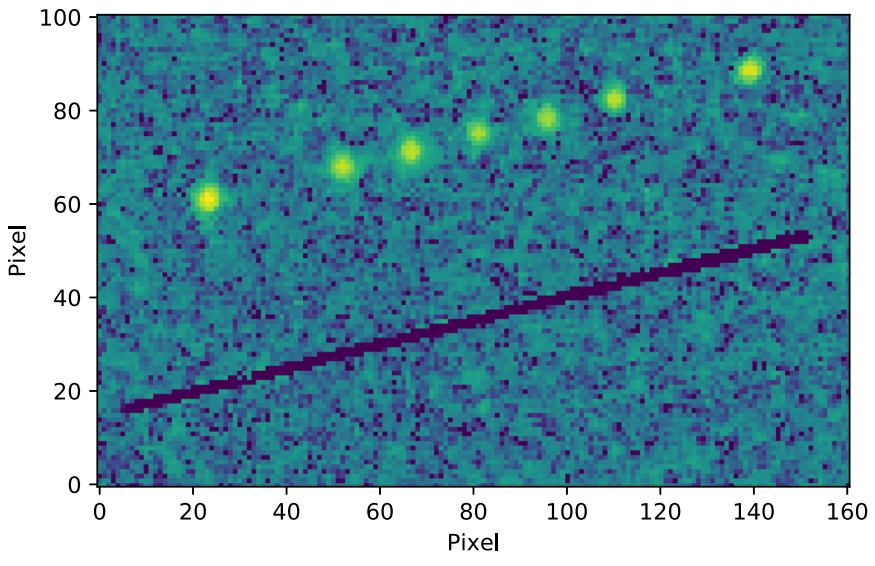

Fig. 7 A composite image with all fibers illuminated acquired with the slit-viewing camera. In this case, the seven PSFs are offset from the slit. The seven PSFs visible on this image are associated to (from left to right): $C_{A}, S_{1}, S_{2}, S_{3}, S_{4}, S_{5}$, and $C_{B}$, respectively. The slit (dark blue) used in this case was the $0.0407^{\prime \prime} \times 1.13^{\prime \prime}$. 


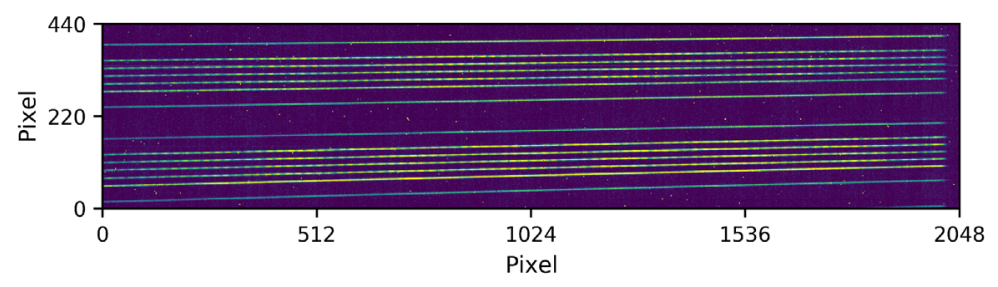

Fig. 8 Two of the nine orders visible on the science detector when configured for $\mathrm{K}$ band observations. Each order of this patchwork of images contained seven spectra. The upper and lower spectra of each order are associated with the two calibration fibers contained in the output connector of the bundle, whereas the five other spectra are associated with the science fibers. This image is a composite image with all fibers illuminated.

\subsection{Expected Performance}

Because the FIU module of KPIC aims to spectrally characterize faint objects with a high resolution $(R>30,000)$, the background seen by the science instrument and the overall throughput are the key properties that need to be understood. In this section, we present our estimations and measurements of these parameters in $\mathrm{K}$ band. Although, the phase I version of the instrument has been designed to allow science observations in $\mathrm{K}$ and $\mathrm{L}$ bands, we have mainly focused our work on the $\mathrm{K}$ band to date. We will start to work in $\mathrm{L}$ bands once most of the other challenges of this project will be overcome.

\subsubsection{Background}

For a fiber-fed spectrograph, the background seen by the science instrument can be decomposed into four components: the background injected into the science fibers of the bundle (sky background and upstream optical elements), the emissivity of the fiber itself, the background emitted between the fiber bundle and the cold stop of NIRSPEC, and the background emitted in the cryostat.

The first component of the background is relatively small. SMFs are extremely efficient at rejecting upstream background from sky, telescope, and FIU because of their relatively small solid angle projected on sky ( $45 \mathrm{mas}^{2}$ in $\mathrm{K}$ band). For reference, the average sky brightness in $\mathrm{K}$ band at zenith during dark times and during a clear night at the summit of Maunakea is equal to $12.6 \mathrm{mag} / \operatorname{arcsec}^{2} .{ }^{39}$ The second component of the background is the emissivity of the fiber itself. We have not quantified this component yet. However, KPIC uses a relatively short (5 m) SMF with very high transmission and with a very small core diameter $(6.5 \mu \mathrm{m})$. We can compute the background emitted based on the energy absorbed by the material of the fiber ( $\leq 0.6 \%$ in $\mathrm{K}$ band). However, it is difficult to estimate the fraction of this background transmitted to the FEU side of the bundle.

The third component of the background is the sum of the emissivities of all the warm optics used to project the light from the fiber onto the NIRSPEC slit (labeled A-G in the Fig. 6). To reduce this component of the background, we use a cold stop, named "FEU stop," located in both filter wheels of NIRSPEC (labeled K on the Fig. 6). These stops have been installed in both wheels to be able to use them with all the NIRSPEC filters available. The stops consist of a hole in a metallic plate, which has been black painted. The hole diameter has been optimized to maximize the SNR seen by the science detector. The radius of the cold stop that maximizes the $\mathrm{SNR}$ " $R_{\max }$ " is

$$
R_{\max } \approx 1.5852 \sigma \approx 0.6731 \mathrm{FWHM},
$$

where $\sigma$ and full width half maximum (FWHM) characterize the Gaussian profile's width. ${ }^{40}$ Using the ratio between the $1 / e^{2}$ diameter and the FWHM $(2 \sqrt{2 \ln (2)} \approx 1.7)$, we can compute the optimal diameter for the cold stop. Because the mode field diameter of the science fibers as well as the effective focal length of the air-spaced doublet and triplet (labeled B and E on Fig. 6) are wavelength dependent, the optimal diameter is also wavelength dependent. Moreover, the 
diameter of the beam at the location of the stop can be affected by the defocus applied to the bundle to optimize the size of the PSF on the slit (see Sec. 4.3). At $2 \mu \mathrm{m}$, we computed the optimal diameter of the stop to be $\approx 7.9 \mathrm{~mm}$. We decided to use a slightly larger cold stops ( $8.1 \mathrm{~mm}$ diameter) because the optical elements of the NIRSPEC calibration unit were never calibrated (as it was not needed) and because the $\mathrm{K}$ and $\mathrm{L}$ bands go beyond $2 \mu \mathrm{m}$. Moreover, the impact of increasing the thermal background slightly is not as severe as restricting the light from the companion.

These stops are used in addition to a blocking filter, which suppresses the science and background light outside of $\mathrm{K}$ band to prevent order overlap. Several filters are available in each filter wheel. Thus far, we have mainly observed in K band (1.9 to $2.5 \mu \mathrm{m})$. In this case, we are using the filter named "thin" located in the first filter wheel. It consists of a substrate of PK-50. This material is a thermal-IR flux blocker opaque for wavelengths $>2.8 \mu \mathrm{m}$. At wavelengths $<1.9 \mu \mathrm{m}$, the background is negligible and the light coming from the target is absorbed by the germanium lens of the air spaced doublet located in the FEU (labeled B in Fig. 6). The few observations made in L band have been made using the broad "KL" filter.

The last component of the background is the internal background of the spectrograph itself. In the case of NIRSPEC, a recently discovered light leak currently adds to the instrument thermal background. $^{41}$

Figure 9 shows the central section of one of the orders visible on a Spec frame [Fig. (9] acquired by pointing at a dark region of the sky (no visible target). The left half of the image is not reduced while the right side is reduced by removing a background acquired during the day (no light injected into the fibers) and correcting for hot pixels. Figure 9(b) shows a vertical line profile of the same order. This profile is the median of the profiles associated to all the wavelengths of the order. The profile computed from the raw data is represented in blue, whereas the profile computed from the reduced data is represented in red.

The two first components of the background (sky and fiber emissivity) are not visible in Fig. 9. If they were visible, we would see small peaks at the locations of the fibers in the red trace. Because we do not use exposure times longer than those used to acquire this data during science observations (599 s), we can consider these two components as negligible in standard observing conditions. More tests need to be done to determine if the conclusion will be the same in $\mathrm{L}$ band, with the telescope pointed at lower elevation or during a night with the moon visible.

The third component, emitted by warm optomechanical components located in the calibration unit of NIRSPEC (see Fig. 6), is visible in the raw data (bright band around the five science fibers) but can be removed efficiently by reducing the data. This contribution to the background is not dependent of the observing conditions but can probably be affected by variation of

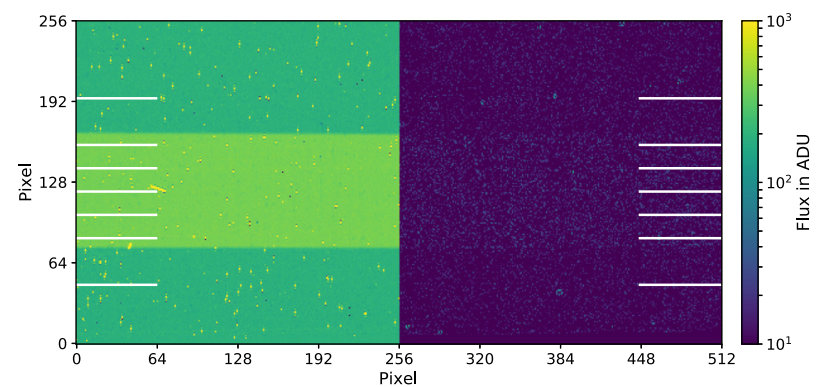

(a)

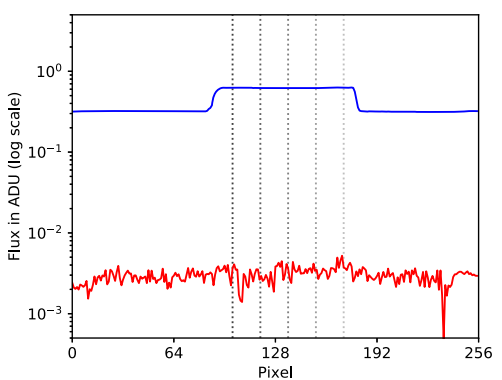

(b)

Fig. 9 (a) Central section of order 33 of a Spec frame acquired with no object in the tracking camera FOV (exposure time $=599 \mathrm{~s}$, Nirspec setup for $\mathrm{K}$ band observation, clear sky, elevation = $54.2 \mathrm{deg}$ no moon). The left side of this image has not been reduced while the right side has been reduced by subtracting a background acquired during the previous night with the same settings and by removing the bad pixels. The horizontal lines of each side indicate the position of the fibers. From top to bottom: $C_{A}, S_{1}, S_{2}, S_{3}, S_{4}, S_{5}, C_{B}$ (see Fig. 3). The slit induced background is visible in the raw data (bright band around the five science fibers). (b) Line profiles across order 33. This profile is the median of the profiles associated to all the wavelengths of the order. The profile computed from the raw data is represented in blue, whereas is computed from the reduced data is represented in red. 
temperature in the $\mathrm{AO}$ room. In this case for an $\mathrm{AO}$ room temperature of $6.1^{\circ} \mathrm{C}$, we measured an average of 0.5 counts per second for the reddest order (order $39-\lambda \in[2440: 2484] \mathrm{nm}$ ) and less than 0.05 counts per second for the bluest order (order $31-\lambda \in[1943: 1977] \mathrm{nm}$ ).

Regarding the last background component, the thermal background of the instrument, it is combined with all the noise and offsets of the science detectors. This component affects the entire image. Visible as an offset below and above the bright green band on the left side of the image presented in Fig. 9, the sum of these effects contributes 180 counts on the reddest side of the detector and 90 counts on the bluest side. As the temperature inside the cryostat is controlled, we can consider this to be constant. As shown in Fig. 9, this component can be removed efficiently. The optical leak discussed previously is not visible is the subimage presented in this paragraph.

\subsubsection{Throughput}

Figure 10 shows the throughput of the overall system (sky to detector included) as a function of the wavelength measured on July 3, 2020, on the star Kappa Andromedae. The throughput is the ratio between the number of photon expected and the number of photons detect by the science detector. Nine orders are visible on this plot. The left one is the 31st order of NIRSPEC, whereas the right one is the 39th order. For this measurement, the light was injected into the second science fiber (labeled $S_{2}$ in Fig. 3). This set of data has been acquired during a clear night. According to the Canada France Hawaii Telescope, the MASS seeing was $\leq 0.2 \operatorname{arc~sec}$ and a DIMM seeing of $\approx 0.6$ arc sec (both measured for a wavelength of $0.5 \mu \mathrm{m}$ ). During this measurement the target was at a 64.5 deg elevation.

It can be seen that the peak throughput in $\mathrm{K}$ band reaches $3 \%$ (well above our $2 \%$ requirement). The data in the figure represent one of the best results achieved to date. Multiple parameters can affect the throughput: seeing, weather, AO performance, and calibration quality. Despite this, over the last few observing runs, we have consistently measured a throughput between $1.5 \%$ and $2.5 \%$ over the $\mathrm{K}$ band.

Neither the Keck II AO system nor the KPIC instrument contains an ADC. As such, the light of the target is slightly dispersed in the focal plane of the input connector of the bundle for nonzero Zenith angles. For this reason, the injection is not uniform across the $\mathrm{K}$ band. In the case presented in Fig. 10, the injection was optimized for a selected wavelength (center of the $\mathrm{K}$ band). This effect is a function of the elevation of the telescope. To optimize the overall throughput of the system for all the wavelengths, an ADC will be deployed in the phase II of the KPIC project.

We also measured the throughput in L band using Kappa Andromeda at the end of the same night. We measured a preliminary throughput of up to $6 \%$ between 3.7 and $3.8 \mu \mathrm{m}$.

The peak throughput measured on-sky of $3.2 \%$ in $\mathrm{K}$ band is consistent with the expected value of 3.4\%, computed assuming 200-nm RMS of residual aberration and taking into account the performance of each of the optics. We have identified several ways to improve the overall

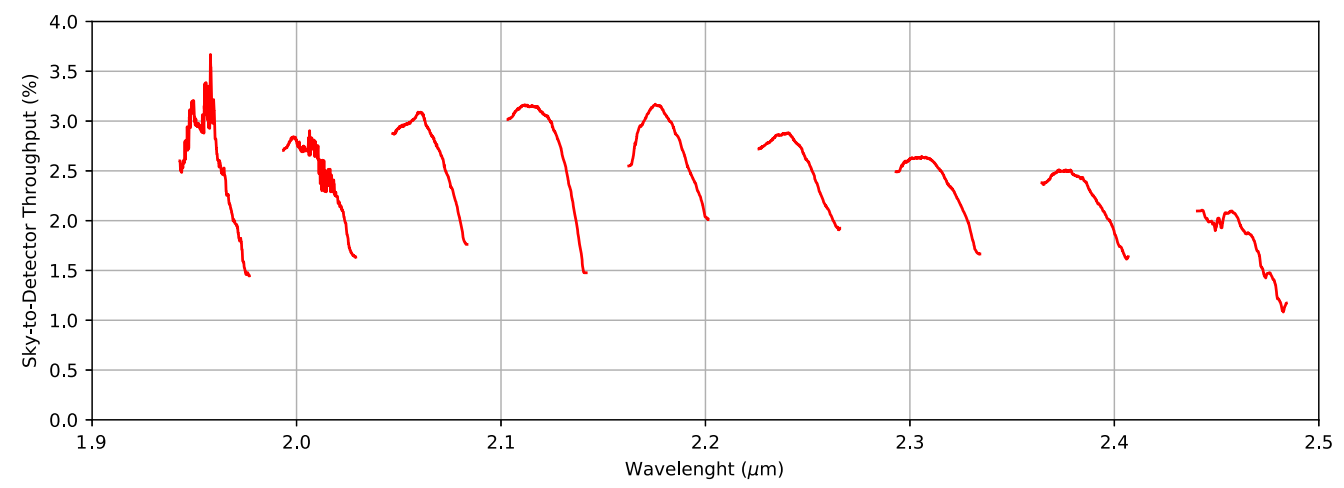

Fig. 10 Throughput as a function of the wavelength from sky-to-detector measured on July 3, 2020, on the star Kappa Andromedae. For this measurement, the light was injected into science fiber $\mathrm{ScF}_{2}$ of the bundle. 
Delorme et al.: Keck Planet Imager and Characterizer: a dedicated single-mode fiber injection unit...

Table 2 Throughput budget computed for the FIU in both $\mathrm{K}$ and $\mathrm{L}$ bands.

\begin{tabular}{lccc}
\hline \hline & & Throughput budget & \\
\hline Components & K-band (\%) & L-band (\%) & Description \\
\hline Sky & 98 & 85 & - \\
Telescope & 90 & 90 & Three reflective optics \\
Keck II AO & 63 & 63 & Seven reflective and one transmissive optics \\
FIU & 77 & 77 & 12 reflective and two transmissive optics \\
Strehl & 72 & 90 & Assuming 200-nm RMS wavefront error \\
Dispersion and pointing loss & 95 & 95 & At 30 deg zenith angle \\
Fiber injection efficiency & 60 & 60 & Keck pupil geometry and NA mismatch \\
Bundle & 95 & 97 & Material absorption and Fresnel reflections \\
FEU + calibration unit & 89 & 89 & Two reflective and five transmissive optics \\
NIRSPEC throughput & 30 & 30 & Diffraction efficiency and optic throughput \\
Filter and background stop & 80 & 80 & From top of atmosphere \\
H2RG quantum efficiency & 95 & 95 & - \\
Total & 3.4 & 3.7 & -
\end{tabular}

performance of the system, which should result in an increase in the quality of the science data in the near future. The following table presents the throughput budget computed for the FIU in both $\mathrm{K}$ and L bands (Table 2).

\section{Calibrations}

Injecting light into an SMF, which subtends a very small solid angle on-sky, requires precise calibration of the instrument. In this section, we describe the various calibrations performed before and during the observations.

\subsection{Fiber Injection Unit Calibrations}

\subsubsection{Science fibers position}

The science fibers are not visible on any sensors, so their positions are unknown at first. The tracking camera is integral to finding the position of the science fibers and optimizing the coupling of a target. By virtue of the dichroic beamsplitter before the tracking camera (M in Fig. 2), J and $\mathrm{H}$ band light can be used to determine the location of a target. To determine the position of the science fibers on the tracking camera, we follow a two-step calibration process. The first one is quick but not highly accurate, whereas the second one is time consuming but very accurate.

The first calibration procedure relies on light to be retro-fed through the peripheral calibration fibers in the bundle. An infrared laser is used and injected into four of the six fibers and emitted from the input connector of the bundle (see Sec. 3.2). The light coming from those fibers is collimated by the focusing OAP of the injection module ( $\mathrm{H}$ in Fig. 2). A fraction of this light is reflected by the dichroic (M) in the direction of the corner cube (T), which reflects the light in the direction of the dichroic. The light transmitted forms an image on the tracking camera composed of four PSFs (one per retro-fed calibration fiber). By retro-feeding an asymmetric pattern of calibration fibers and by knowing the geometry of the bundle, we can determine the position of the other fibers (science fibers) in the input connector of the bundle. Figure 11 shows the

J. Astron. Telesc. Instrum. Syst. $\quad$ 035006-14 Jul-Sep 2021 • Vol. 7(3) 


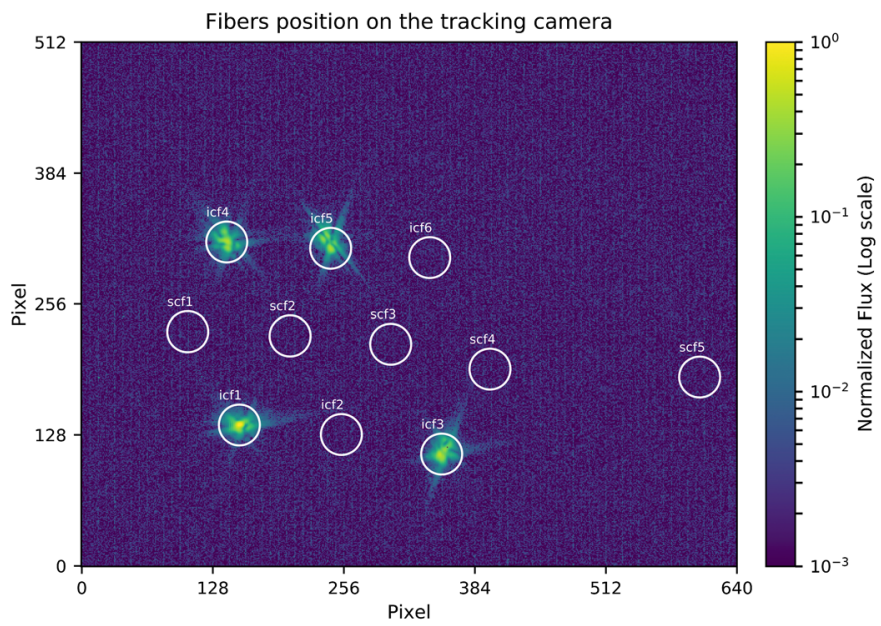

Fig. 11 Output figure automatically generated by the algorithm used to roughly determine the position of each fiber of the bundle. The "X" shape of the PSFs is due to the corner cube.

output automatically generated by the algorithm used to perform this calibration procedure. It takes only a few seconds to get a result but the estimated positions are not highly accurate (usually a couple of pixels) for multiple reasons: the shape of the PSFs for the four calibration fibers is altered by the OAP and the corner cube, which makes the position measurement of these fibers inaccurate, even if the geometry of the bundle is precisely known, we do not take into account field distortions at the detector, and the corner cube is not perfect so the incident and reflected beams are not parallel, to name a few.

To maximize the throughput of the instrument, we use a second calibration procedure based on a direct measurement of the light injected into the fibers. For this step, we turn on the calibration source of the Keck II AO bench to create a PSF on the tracking camera and then use the TTM of the FIU (labeled L on Fig. 2) to align this PSF with one of the science fibers. Then, we scan the TTM across the expected location of the fiber and record the flux on the slit viewing camera of NIRSPEC, Scam (see Fig. 6) as well as the tracking camera. The SCAM images are used to measure the flux injected into the science fiber while the tracking camera images are used to measure the flux variation of the light source and track the PSF position associated with each flux measurement. In this way, we can build and injection map through the fiber and by fitting the peak, we can determine the TTM position of optimum coupling very accurately and the corresponding PSF position that yields this optimum alignment on the tracking camera at the same time. Figure 12(a) shows the injection map obtained by performing such a scan. The position of each pixel of this injection map corresponds to a position of the PSF on the tracking camera, and the value associated with each pixel is the flux measured using Scam normalized by the flux of the calibration source measured by the tracking camera. This calibration method is time consuming. We are currently limited by the minimum exposure time of the Scam detector

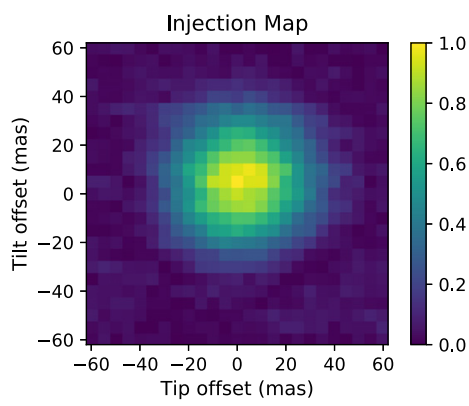

(a)

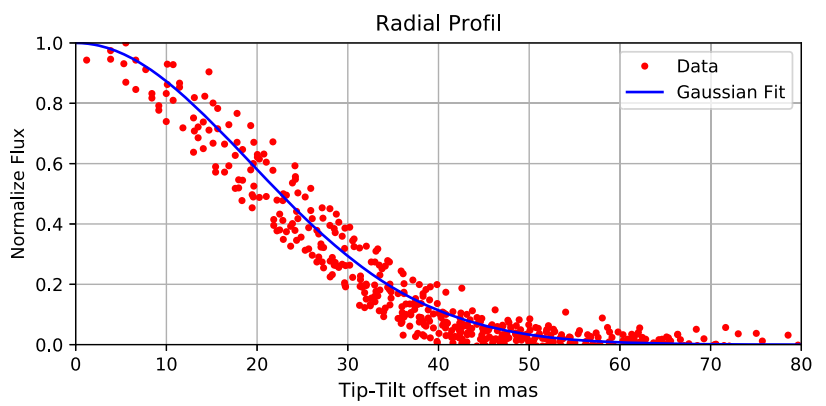

(b)

Fig. 12 (a) Injection map obtained by performing a $25 \times 25$ grid scan. The pitch of the grid is 5 mas in both direction. (b) Radial profile computed from the injection map. 
$(\approx 600 \mathrm{~ms})$ and its read-out $(\approx 1200 \mathrm{~ms})$. We tried multiple scan patterns to reduce the time needed to perform this calibration but for the moment, the grid scans are the most accurate and reliable. As shown by the injection map radial profile shown in Fig. 12(b), knowledge of the position of each fiber is critical if we want to maximize throughput. An offset of one pixel on the tracking camera (8.06 mas) corresponds to a loss of $10 \%$ of throughput, two pixels (16.12 mas) corresponds to a loss of $30 \%$, and three pixels (24.18 mas) to a loss of 55\%. An offset of 50 mas that corresponds to the angular resolution of the Keck telescopes at $2 \mu \mathrm{m}$ corresponds to a loss of $97 \%$ of the throughput. This is why we take the time to perform these calibrations properly and why we perform theses calibration before each science night even if the bundle has not been disconnected between two observations.

During the calibrations, the PSFs in H, K, and L bands are superimposed. As neither the Keck II AO system nor the first phase of the KPIC project contains an atmospheric dispersion corrector, when on-sky the PSFs of the target in $\mathrm{H}, \mathrm{K}$, and L bands are superimposed only when observing at Zenith. Therefore, when observing away from Zenith, the location of the PSF on the tracking camera, which operates in $\mathrm{H}$ band, will not correspond to the location of the $\mathrm{K}$ and $\mathrm{L}$ band PSFs in the focal plane of the injection OAP. As such, an additional step is taken during the acquisition sequence, whereby the PSF is offset with the TTM along the elevation axis by the calculated offset expected from the differential atmospheric refraction between the $\mathrm{H}$ and science bands ( $\mathrm{K}$ or $\mathrm{L}$ ). This offset is a function of the elevation of the target and the refractive index of the air versus wavelength. To compute the latter, we use an analytical approximation of the refractive index that depends on the temperature, pressure, and relative humidity of the air above the primary mirror. ${ }^{42}$ The tracking loop used to align the target with the fiber computes this value and applies the offset at each iteration of the loop (see Sec. 5).

\subsubsection{Tracking camera}

The calibration of the tracking camera is critical because we use it as a reference to inject the light of the target of interest into one of the science fibers. Key to calibrating the camera is understanding the plate scale, the orientation, and the field distortion map. We use two different methods to calibrate the tracking camera. The first one can be performed during the day using NIRC2 as a reference. The plate scale, the orientation, and the distortion map of this detector is regularly calibrated and can be considered as reliable. Using a dichroic like the KPIC-pick off (labeled A on Fig. 2), we can image the PSF of the calibration unit of the Keck II AO bench on both NIRC2 and the tracking camera at the same time. We translate the calibration source in both the $X$ and $Y$ axes and acquire images for each position with both detectors. We compute the position of the PSF in each image. A set of two positions can be used to determine the plate scale of the tracking camera, and a set of three positions forming an asymmetric pattern can be used to determine the orientation of the tracking camera with respect to NIRC2. The more sets of data available, the more accurate the plate scale and the orientation. The variation as a function of $x / y$ on the detector of plate scale and orientation between those sets of data is used to compute the distortion map of the tracking camera. To model the distortion in $X$ and $Y$ on the detector, we use the methodology of Service et al. $2016^{43}$ and use a fifth-order bivariate Legendre polynomial. A second calibration method performed during the night is routinely used to refine this. This method consists of observing an object with multiple components (a binary star for example). Because the astrometry of such an object (position angle and separation) is stable during the calibration, we can use images of this object acquired on different parts of the detector to calibrate the distortion map of the tracking camera. To move the object around the detector, we steer the TTM in the FIU. Indeed, in the presence of local field distortions, the position angle and separation vary across the detector. The average separation of this target can be used to determine the plate scale and the orientation of the detector. To mitigate orbital motion uncertainties of the system observed to perform this calibration, we image the system with NIRC2 and the Cred2 in parallel. We also tried to observe known fields of stars, such as M92. However, the FOV of the tracking camera $(4.5 \times 5.5$ arc sec $)$ is too narrow to image many bright stars at the same time $(\mathrm{H}-$ $\operatorname{mag} \leq 10)$.

The offsets computed from the calibrations outlined above are applied to the tracking loop (see Sec. 5) to align the science target with the selected science fiber. We verify at the beginning 
of each run that we can align the PSF with a subpixel resolution to confirm the validity of the tracking camera calibrations.

\subsubsection{Coupling optimization}

To optimize the coupling, we must reduce the wavefront error in the system to maximize the image quality in the focal plane of the input connector of the fiber bundle. However, this plane is not easily accessible and cannot be imaged with a detector so we cannot use any standard focal plane wavefront sensing strategy in this case. The solution found to overcome this problem is to use two calibration procedures. The first one is used to optimize the image quality on the tracking camera, whereas the second one is used to calibrate the noncommon path aberrations (NCPA) between the two paths of the FIU (tracking camera and injection module).

To optimize the image quality on the tracking camera, we use a phase diversity algorithm called "image sharpening." This is used in the calibration procedure for some of the Keck facility instruments. This procedure can be run before each science night as it takes only a few minutes to complete. Indeed, even if the version used is not fully automated yet, we never start from a very deformed PSF. We usually start with $<100 \mathrm{~nm}$ RMS of wavefront aberration in the system and consistently reach wavefront errors $<20 \mathrm{~nm}$ RMS using this procedure.

Once the wavefront error is reduced to maximize the image quality on the tracking camera, we use a Zernike optimization strategy that measures the flux transmitted through the fiber bundle to indirectly reduce the NCPA between the two paths of the FIU. We first select a Zernike mode (we usually start with the defocus). Then, we scan this mode in amplitude using the Keck AO DM. For each amplitude, we perform a tip-tilt scan to build an injection map (see Sec. 4.1.1). This is done in case the PSF moves laterally while being defocus (i.e., in case there is crosscoupling between modes). By fitting a two-dimensional Gaussian to each injection map, we obtain the optimal injection for each Zernike amplitude. The optimal amplitude for the selected Zernike mode is determined by fitting a second-order polynomial on the optimal injection values measured for each amplitude of the selected Zernike mode. We then apply this value to the DM and continue the calibration procedure by scanning the next Zernike mode. This calibration procedure is completed once all the Zernike modes selected have been scanned. Because we currently only scan one mode at a time, we assume the modes in the system are orthogonal, which is a reasonable assumption. In addition, most of the NCPA is in the lowest order modes so we typically do not need to scan beyond 15 Zernike modes.

Figure 13 shows some of the injection maps obtained while optimizing the defocus for the first three science fibers. Each row is associated to a fiber (top row to $S_{1}$, central row to $S_{2}$, and bottom row to $S_{3}$ ) and each column to a defocus amplitude (from left to right: $-180,-90,0$, +90 , and $+180 \mathrm{~nm}$ RMS). In this figure, we can see that the NCPA is not the same for each fiber of the bundle. Indeed, the elongation in the injection maps hints at the presence of astigmatism in the PSF at the location of some of the fibers. Because this calibration is time consuming and we mainly use the second science fiber of the bundle on sky, we only run the calibration for this fiber routinely.

Using this calibration procedure, we have been able to improve the injection by $\sim 13 \%$. This result has been obtained by scanning eight Zernike modes (Noll index $\in[4: 11]$ ). The results presented in this paragraph have been obtained by connecting the bundle to a photodetector using a simple optomechanical assembly. The use of the photodetector allows us to run the calibration faster but prevents us from running the calibration when the bundle is connected to NIRSPEC. In the future, we aim to be able to run this calibration using Scam and/or Spec. In addition, we need to improve the algorithm to converge faster. We also want to characterize the NCPA and determine how it has evolved over time to determine how often this calibration has to be performed.

\subsection{Fiber Extraction Unit Calibrations}

NIRSPEC can be used on both Nasmyth platforms of the Keck II telescope. As such, the bundle has to be reconnected to the FEU each time the instrument is transported. Even if the repositioning of the bundle (focus and orientation) is repeatable and the FEU stable over time, we perform 

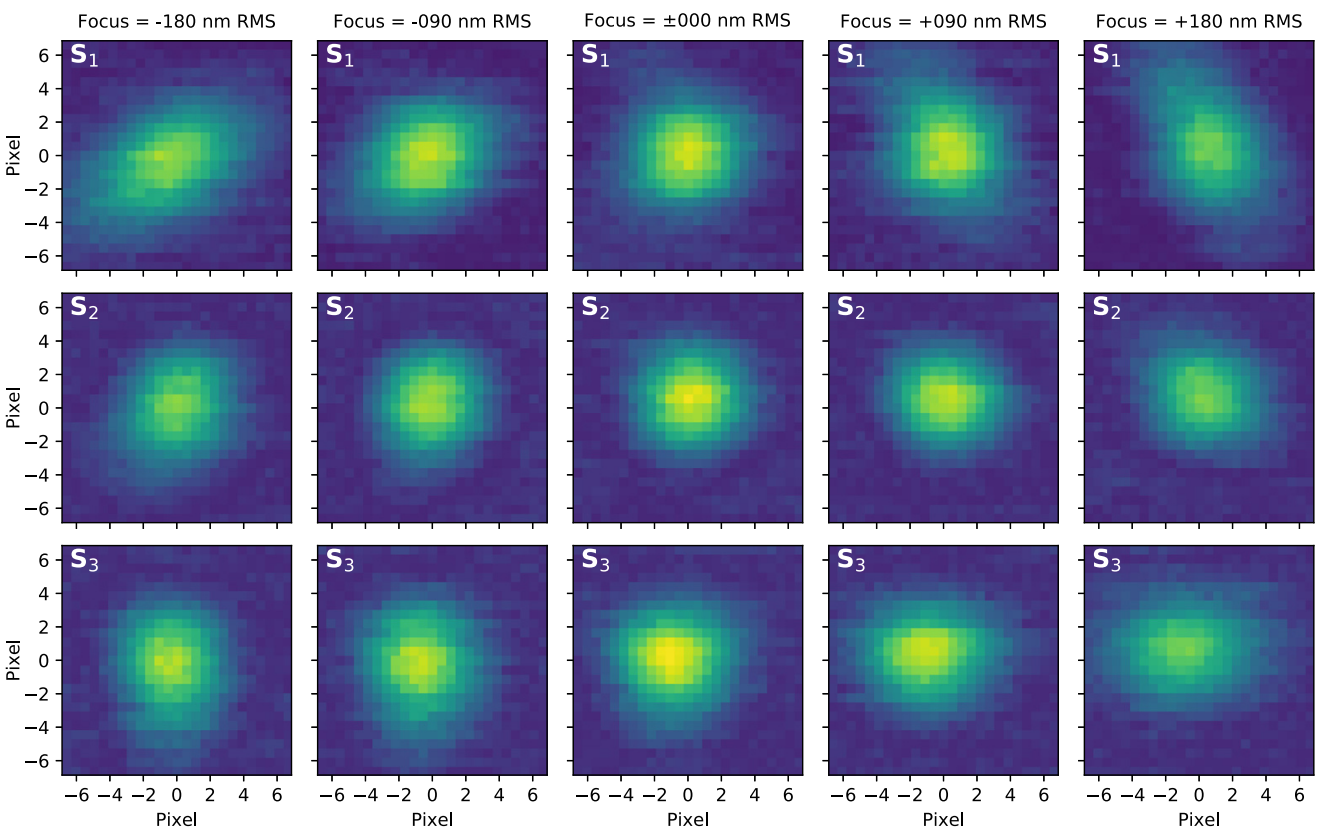

Fig. 13 Measured injection maps from data collected on three fibers: $S_{1}$ (top row), $S_{2}$ (center row), $\mathrm{S}_{3}$ (bottom row), and five defocus amplitudes centered on the initial defocus. From left to right: $-180,90,0,+90$, and $+180 \mathrm{~nm}$ RMS. The flux of the injection map has been normalized by the maximum of all the injection maps measured for those three fibers (63 total).

basic calibrations of this module before each science night to optimize the performance of the instrument.

The first calibration is used to coalign the fibers contained in the output connector of the bundle with the slit selected for the observation. To move the fibers with respect to the slit, we use the FEU-TTM (labeled C in Fig. 6). To rotate the fibers with respect to the slit, we use the rotator mirror contained inside the NIRSPEC cryostat. Usually, the rotator does not need to be rotated by more than a degree, because the bundle was clocked to approximately the right orientation by hand initially.

The second calibration is used to coalign the beam coming from the fiber bundle with the stop used to reduce the background (see Sec. 3.4). This stop is in the pupil plane of the fore-optics. To move the beam with respect to the stop, we translate the bundle in the planes perpendicular to the optical axis using two of the motorized translation stages contained in the FEU (see Sec. 3.3).

The third calibration is used to focus the bundle and adjust the size of the PSFs coming from each fiber of the bundle on the slit. Because NIRSPEC has not been designed to be diffractionlimited, the PSF is smaller than the slit when the bundle is perfectly in focus. In this case, the spectrum acquired by the science detector of NIRSPEC is undersampled. To overcome this problem, we have the capability to defocus the bundle to optimize the sampling of the science data. We adjust the focus of the bundle using one of the motorized translation stages contained in the FEU (see Sec. 3.3). At the shortest observed wavelength in a given band, we adjust the focus of the bundle to get 2.4 pixels/FWHM and this produces an $R \sim 35 \mathrm{k}$.

These three calibration procedures are relatively rapid to perform due to the stability of the FEU (usually less than one hour). The adjustments between two consecutive nights are typically minor.

\subsection{NIRSPEC Calibrations}

KPIC uses NIRSPEC in nonstandard ways, so additional calibration steps on top of the standard ones are necessary. The days before the observation, we take the standard backgrounds and darks with multiple instrument set ups we are likely to use during the science nights. We have the possibility to take extra calibration data after the night if needed but calibration data acquired 
before the science night can be used to quickly reduce the data during the night to verify if everything is working as expected.

During the days before the observation, a wavelength solution calibration of the science detector is performed using NIRSPEC's calibration sources (see Fig. 6). The different arc lamp sources available in this unit have known emission spectra, which enable wavelength calibration of the instrument. The calibration unit also contains an extended white light source. We use this source to acquire flats for every configuration we plan to use during the night.

The next step of the NIRSPEC calibrations consists on determining where the traces associated with each fiber in the bundle are located on the science detector. The position of these spectra must be known to extract the spectral information of each target observed. In the case of a bright object, the location of the spectra is obvious but this is not the case for faint objects, and so determining where to look for the traces with a calibration source off-sky can be beneficial. To perform this calibration, we set up NIRSPEC, align the fibers of the output connector with the slit using the position determined previously (see Sec. 4.2), and inject the light of the calibration source of the Keck II AO bench in each science fiber, one at a time. For each science fiber, we acquire an image with the science detector. Because the calibration source of the Keck II AO bench is bright and its emission spectra continuous, we can extract the position of the traces associated with each science fiber, across all the orders visible on the detector. Because the position of the spectrum on the science detector and the wavelength solution are dependent on the position of all the optomechanical elements located downstream of the bundle, we need to perform this calibration for each instrument configuration we plan to use during the science nights.

When we observe a target with at least one bright component ( $\mathrm{K}$ or L magnitude $<10$ ), we repeat this calibration on-sky. By acquiring such a data set on-sky, we can insure that our calibrations were not affected by long-term thermal variations as the calibration is taken close to when the science data are acquired. In addition, we can observe the target of interest and perform the calibration by keeping all the optomechanical elements located downstream of the bundle fixed, which yields a superior wavelength solution. Finally, the data collected on the main star of the system can be used in the reduction of the science data.

\section{Science Data Acquisition}

The FIU can be used to observe different kinds of targets. In this section, we only described the data acquisition procedure followed to acquire high-resolution spectra of exoplanets and brown dwarfs. In most cases, we cannot image the exoplanet using the tracking camera during an observation. So, to blindly align the PSF of an exoplanet with one of the science fibers of the bundle, we need to have an accurate knowledge of the astrometry of the system being observed (position angle and separation). For companions with well-characterized orbits, such as the HR 8799 planets for example, ${ }^{44}$ we can use the predicted relative astrometry or use data collected during the most recent observation to measure the position. To predict the position of a planet, we use the algorithm used by the website. ${ }^{45}$ If the information is not available or not reliable, we can observe the target with NIRC2 at the beginning of our observing run to measure the astrometric parameters of the companion.

During the observation, the first step of the acquisition sequence consists of aligning the star with the selected science fiber. To achieve this, we use a tracking loop. Its goal is to align the science target or its host star with a science fiber by controlling the TTM in the FIU (labeled L, on Fig. 2). It uses as inputs the image from the tracking camera as well as multiple parameters provided by the telescope, the calibration procedures as well as the user.

The algorithm uses tracking camera images to determine the position of the host star on the detector. Using the position of the science fibers determined during the calibration (see Sec. 4.1), the algorithm determines the FIU-TTM offset needed and applies the command. As described in Sec. 4.1, we need to take into account the atmospheric dispersion. This effect is automatically compensated by the tracking loop at each iteration. For a given Zenith angle, temperature, pressure, and relative humidity, the offset in the differential atmospheric refraction between $\mathrm{H}$, where the camera tracks and $\mathrm{K}$, where science is conducted, is calculated and applied along the elevation axis (axis of refraction). At this point, we usually acquire data using the science detector of 


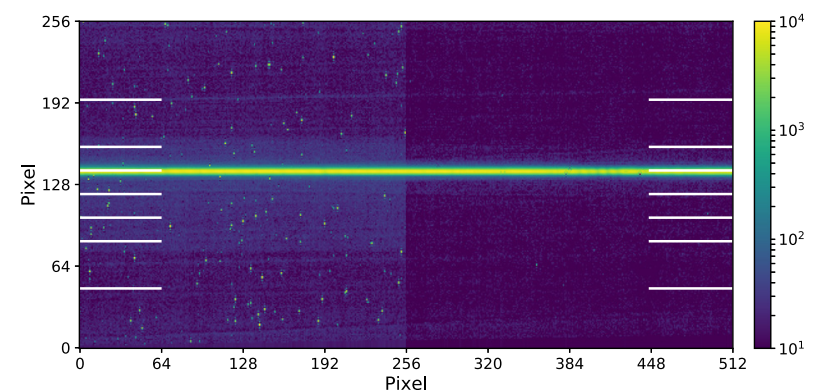

(a)

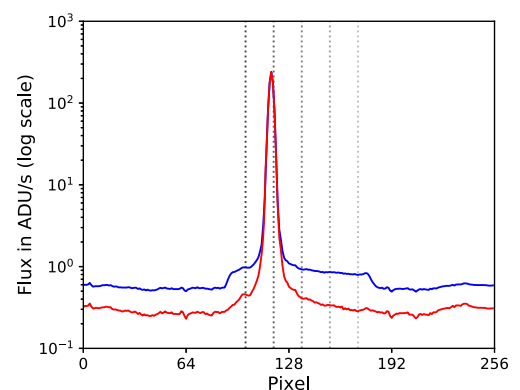

(b)

Fig. 14 (a) Central section of order 33 of a Spec frame acquired with the star of the HR 7672 system align with the second science fiber (exposure time $=29.5 \mathrm{~s}$ ). The left side of this image has not been reduced, whereas the right side has been reduced by subtracting the background and removing the bad pixels. The horizontal lines of each side indicate the position of the fibers. From top to bottom: $\mathrm{C}_{A}, \mathrm{~S}_{1}, \mathrm{~S}_{2}, \mathrm{~S}_{3}, \mathrm{~S}_{4}, \mathrm{~S}_{5}, \mathrm{C}_{B}$ (see Fig. 3). (b) Profiles of order 33. This profile is the median of the profiles associated across all the wavelengths of the order. The profile computed from the raw data is represented in blue, whereas the line profile computed from the reduced data is represented in red.

NIRSPEC on the host. These data are used to measure the throughput of the instrument and to reduce the science data acquired later in this sequence. Figure 14 shows data acquired with the star of the HR7672 system aligned with the second science fiber (labeled $S_{2}$ on Fig. 3) on July 3, 2020. Figure 14(a) shows the central section of the order $33(\lambda \in[2047: 2083] \mathrm{nm})$ before and after reduction. Figure 14(b) shows the median line profile of this order. The blue curve is the line profile computed from the raw data, whereas the red one is computed from the reduced data. The spectra of the star, aligned with the second science fiber of the bundle, is clearly visible on both the Spec frame and the graph presented by this figure.

After aligning the star with the selected science fiber and the spectral data collected, we use the relative astrometry of the companion to offset the star to align the science target with the fiber. This offset is applied by providing the astrometric parameters of the target (position angle and separation) to the tracking loop. After correcting the astrometric parameters to take into account the field distortion of the tracking camera, the tracking loop computes the offset needed and sends a command to the FIU-TTM to apply the offset. We verify at the beginning of each run that we can apply such offsets with a subpixel accuracy by observing several binary stars with both components visible on the tracking camera. At this point, the integration on the science target (the companion) begins. In the case of faint targets ( $K$ or $L$ magnitude $\geq 13$ ), we acquire multiple $10 \mathrm{~min}$ exposure frames with the science detector of NIRSPEC. Figure 15(a) shows a section of order 33 extracted from one of the science frames acquired the HR7672b system aligned with the second science fiber. Figure 15(b) shows the median profiles of this order. The blue curve is the profile computed from the raw data, whereas the red one is computed from the reduced data. Three peaks are visible in the line profile, which indicate the position of the fiber traces. The one associated with the second science fiber contains a combination of the light coming from the science target, the speckle field of the star, and the multiple backgrounds described in Sec. 3.5.1. The line associated with the third and fourth science fiber contains the light of the speckle field of the star and the multiple backgrounds (see Sec. 3.5.1). We cannot adjust the intensity of the light coming from the speckle field of the star in the science fiber aligned with the companion as the separation between the companion and its host start is constant. However, it can be adjusted for the other fiber by moving the star around the science target using the rotator of the telescope. The offset applied to the rotator is automatically taken into account by the tracking loop.

During the observation of a faint target, we regularly acquire data with the star of the system aligned with the selected fiber (every hour or six science frames) to get enough calibration data and to make sure the injection into the fiber is high and stable by measuring the throughput of the system. 


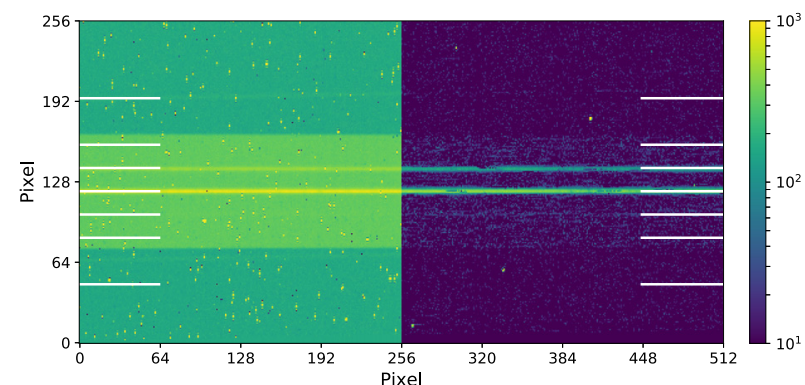

(a)

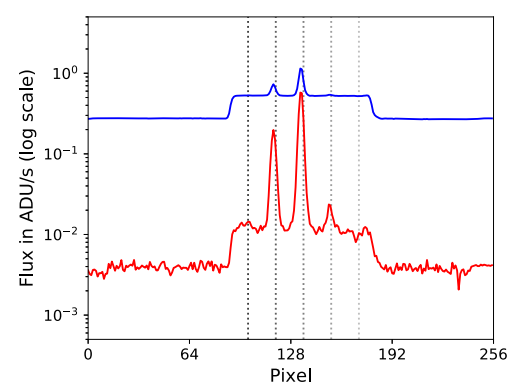

(b)

Fig. 15 (a) Central section of the order 33 of a Spec frame acquired with the companion of the HR 7672 system align with the second science fiber (exposure time $=599 \mathrm{~s}$ ). The left side of this image has not been reduced, whereas the right side has been reduced by subtracting the background and removing the bad pixel. The horizontal lines of each side indicate the position of the fibers. From top to bottom: $\mathrm{C}_{A}, \mathrm{~S}_{1}, \mathrm{~S}_{2}, \mathrm{~S}_{3}, \mathrm{~S}_{4}, \mathrm{~S}_{5}, \mathrm{C}_{B}$ (see Fig. 3). (b) Profiles of the order 33 computed by averaging the profile associated to each wavelength of the order. The profile computed from the raw data is represented in blue, whereas the profile computed from the reduced data is represented in red.

\section{On Sky Performance and First Results}

To demonstrate the science capabilities of the system, we observed HR 7672 B on September 28, 2020, a bright ( $K=13 \mathrm{mag}$ ) early L-type brown dwarf that is expected to have a similar spectral signature as many of our more challenging targets. ${ }^{26}$ Residing 0.64 arc sec from the star at the epoch of observation, ${ }^{46}$ the companion also resides at comparable separations from the star as our exoplanet targets. We obtained ten 600-s exposures of the brown dwarf placed on science fiber $\mathrm{S}_{2}$, resulting in a total integration time of $100 \mathrm{~min}$. The data were reduced using a preliminary version of the KPIC data reduction pipeline. The thermal background of the instrument was subtracted using $8 \mathrm{~h}$ of backgrounds taken during the day with the same instrument setup. The A0 standard star ups Her was observed at the start of the night to calibrate the position and width of all of the fiber traces. We used this trace information to extract spectra from all of the

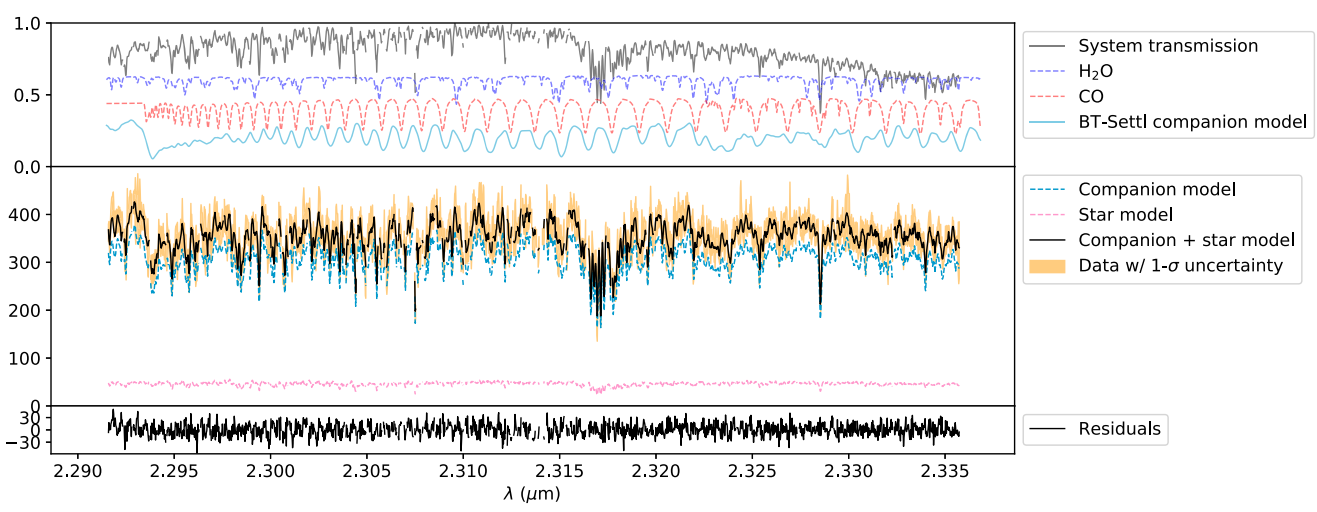

Fig. 16 Spectrum of HR 7672 B (37th NIRSPEC order). The middle panel includes the extracted spectrum of the science fiber including its 1- $\sigma$ uncertainties (orange contours). The spectra were high-pass filtered but plotted with an offset corresponding to the continuum median value. The best-fit forward model of the data is shown as a black line. Because the spectrum of the companion is contaminated by the glare of the star, we jointly fit an on-axis starlight spectrum with a model of the planet. Both components of the model, which include telluric lines, are shown separately as dashed lines. The companion is modeled with a BT-SETTL template assuming a $1800 \mathrm{~K}$ temperature and a $\log (g)=5.0$ surface gravity. The top panel features spectral templates that can be used to identify spectral lines in the data. The system transmission includes: the transmission of the atmosphere and the instrument. The BT-Settl model includes spin broadening of the companion, whereas the molecular template is only broadened to the resolution of the instrument. The lower panel shows the residuals of the fit. 
science fibers of the HR 7672 exposures, resulting in on-axis observations of both HR 7672 A and $\mathrm{B}$ on $\mathrm{S}_{2}$. The extracted spectra were then averaged in time, removing bad pixels due to cosmic ray contamination. The M-giant HIP 81497 was observed in each fiber to compute the wavelength solution of each fiber. The A0 star zet Aql was observed immediately before the HR 7672 observations to measure the total end-to-end spectral response, including the telluric absorption profile.

The spectral response, wavelength solution, on-axis observations of $S_{2}$, and planetary atmosphere models were used to construct forward models of the on-axis spectra of the brown dwarf companion. We used a BT-SETTL atmosphere model with an effective temperature of $1800 \mathrm{~K}$ and a $\log (g)=5.0$ for the planet spectrum. ${ }^{47}$ We varied the radial velocity (RV) of the planet, rotational broadening, and overall flux of the planet in fitting the forward model to the data. A more detailed description of the data extraction and fitting technique will be discussed in a subsequent paper. Figure 16 shows the best-fit model and data from order 37 of the spectrum of HR $7672 \mathrm{~B}$. The best-fit forward model matches the data very well, as can be seen in visual inspection. To better visualize the detection, we show the CCF detection of HR 7672 B in the top row of Fig. 17. There is a clear detection of the brown dwarf and is consistent with previous $R \sim 1400$ spectra at the same wavelength. ${ }^{26}$ The posterior distributions for the RV of the planet and its rotational broadening are shown in the bottom row of Fig. 17. The vsin(i) measured is (42.6 \pm $0.8) \mathrm{km} / \mathrm{s}$ and is the first detection of rotational broadening for this companion. The detection and spin measurement of a companion just 0.64 arc sec from its host star demonstrates the HDC capabilities of KPIC.

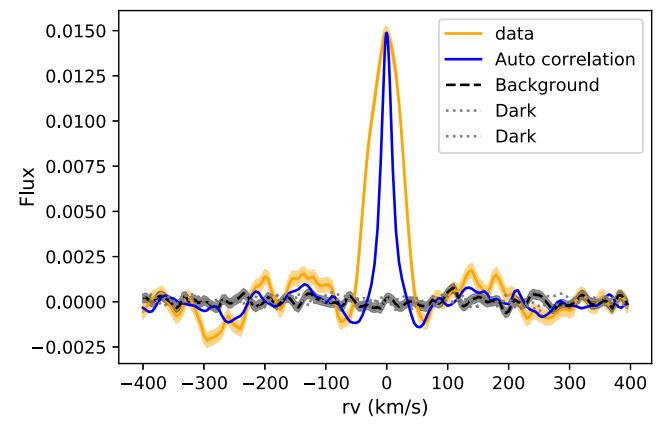

(a)

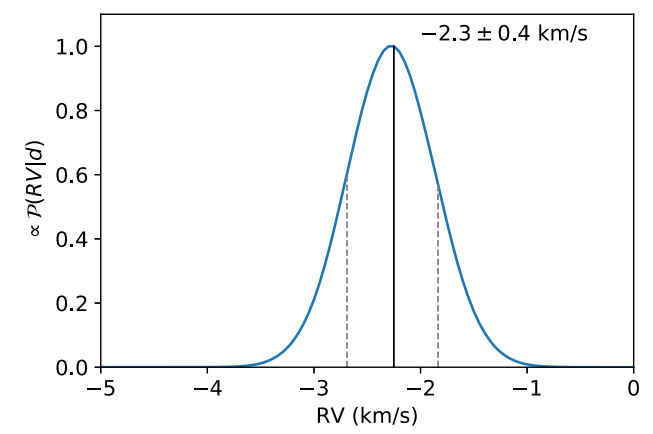

(c)

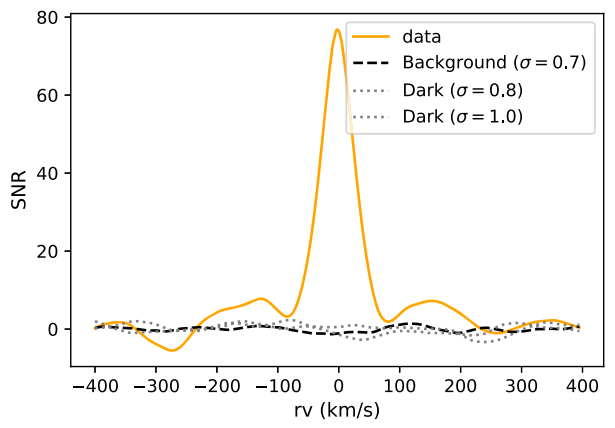

(b)

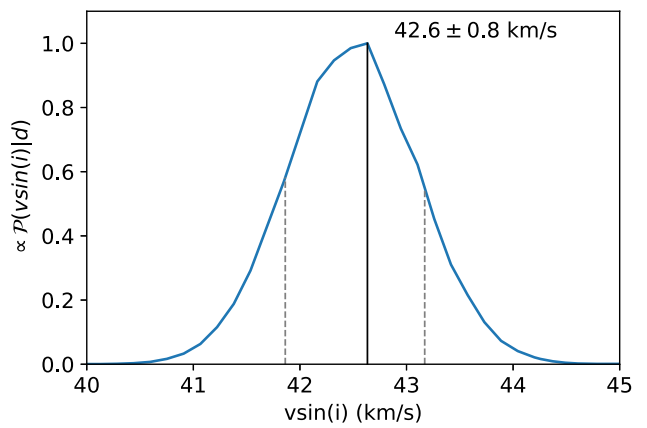

(d)

Fig. 17 Detection of HR 7672 B using the four reddest NIRSPEC orders (Order 36-39). (a) A type a cross correlation analysis. It is calculated using the forward model framework illustrated in Fig. 16 as the estimated flux and associated uncertainty of the brown-dwarf companion as a function of the RV shift of the model assuming a null spin for the companion. Fictitious fibers that only include background and dark flux are also analyzed with the same model as a way to sample the noise. The top right panel is the signal to noise $(\mathrm{S} / \mathrm{N})$ of the detection as a function of $\mathrm{RV}$ assuming the best fit $\operatorname{spin}(v \sin (i)=42.6 \mathrm{~km} / \mathrm{s})$ for the model. The $\mathrm{S} / \mathrm{N}$ is calculated as the estimated amplitude of the companion divided by its estimated uncertainty featured in (b). (c), (d) The marginalized posteriors for the RV and the spin of the companion, respectively. The estimated RV of the companion is consistent with the prediction from relative astrometry measurements of the companion and the RV of the host star. 


\section{Conclusion}

The FIU, part of the KPIC demonstrator, is a powerful tool to acquire high spectral resolution data on faint objects surrounding stars, faint isolated objects, and close binaries. Deployed at the summit of Maunakea in the fall 2018, the FIU has completed commissioning and has begun regular science operations earlier this year. Numerous known stellar systems have been imaged already and science data acquired. These data, currently being reduced and analyzed, will be published in subsequent papers. After multiple months of science exploitation, the second phase of the project will be deployed to improve the overall performance of the instrument. The hardware, software, and observing strategies developed, tested, and used by both phases of this project will help to prepare future instruments on both ground- and space-based telescopes.

\section{Acknowledgments}

This work was supported by the Heising-Simons Foundation through Grant No. 2019-1312. It has been previously submitted as an SPIE proceeding. ${ }^{48} \mathrm{~W}$. M. Keck Observatory is operated as a scientific partnership among the California Institute of Technology, the University of California, and the National Aeronautics and Space Administration (NASA). The observatory was made possible by the generous financial support of the W. M. Keck Foundation. The authors wish to recognize and acknowledge the very significant cultural role and reverence that the summit of Mauna Kea has always had within the indigenous Hawaiian community. We are most fortunate to have the opportunity to conduct observations from this mountain.

\section{References}

1. A. Wolszczan and D. A. Frail, "A planetary system around the millisecond pulsar PSR1257 + 12," Nature 355, 145-147 (1992).

2. M. Mayor and D. Queloz, "A Jupiter-mass companion to a solar-type star," Nature 378, 355-359 (1995).

3. D. Charbonneau et al., "An upper limit on the reflected light from the planet orbiting the star $\tau$ bootis," Astrophys. J. 522, L145-L148 (1999).

4. C. Moutou et al., "Search for spectroscopical signatures of transiting HD 209458b's exosphere,” Astron. Astrophys. 371, 260-266 (2001).

5. I. S. McLean et al., "NIRSPEC: a near-infrared cross-dispersed echelle spectrograph for the Keck II telescope," Proc. SPIE 2475, 350-358 (1995).

6. H.-U. Kaeufl et al., "CRIRES: a high-resolution infrared spectrograph for ESO's VLT," Proc. SPIE 5492, 1218-1227 (2004).

7. I. A. G. Snellen et al., "The orbital motion, absolute mass and high-altitude winds of exoplanet hd 209458b," Nature 465(7301), 1049-1051 (2010).

8. G. Rousset et al., "Status of the VLT Nasmyth adaptive optics system (NAOS)," Proc. SPIE 4007, 72-81 (2000).

9. R. Lenzen et al., "CONICA: the high-resolution near-infrared camera for the ESO VLT," Proc. SPIE 3354, 606-614 (1998).

10. G. Chauvin et al., "A giant planet candidate near a young brown dwarf," Astron. Astrophys. 425, L29-L32 (2004).

11. C. Marois et al., "Direct imaging of multiple planets orbiting the star HR 8799," Science 322(5906), 1348-1352 (2008).

12. J. Larkin et al., "OSIRIS: a diffraction limited integral field spectrograph for Keck," 50, 362-364 (2006).

13. M. F. Skrutskie et al., "The Large Binocular Telescope mid-infrared camera (LMIRcam): final design and status," Proc. SPIE 7735, 1368-1378 (2010).

14. B. R. Oppenheimer et al., "Project 1640: the world's first ExAO coronagraphic hyperspectral imager for comparative planetary science," Proc. SPIE 8447, 844720 (2012).

15. J. L. Beuzit et al., "SPHERE: the exoplanet imager for the Very Large Telescope," Astron. Astrophys. 631, A155 (2019). 
16. B. Macintosh et al., "First light of the Gemini Planet Imager," Proc. Natl. Acad. Sci. U. S. A. 111, 12661-12666 (2014).

17. T. D. Groff et al., "Laboratory testing and performance verification of the CHARIS integral field spectrograph,” Proc. SPIE 9908, 990800 (2016).

18. I. A. G. Snellen et al., "Fast spin of the young extrasolar planet $\beta$ Pictoris b," Nature 509, 63-65 (2014).

19. I. Snellen et al., "Combining high-dispersion spectroscopy with high contrast imaging: probing rocky planets around our nearest neighbors," Astron. Astrophys. 576, A59 (2015).

20. J. Wang et al., "Observing exoplanets with high dispersion coronagraphy. I. The scientific potential of current and next-generation large ground and space telescopes," Astron. J. 153, 183 (2017).

21. D. Mawet et al., "Observing exoplanets with high-dispersion coronagraphy. II. Demonstration of an active single-mode fiber injection unit," Astrophys. J. 838, 92 (2017).

22. H. Kawahara et al., "Spectroscopic coronagraphy for planetary radial velocimetry of exoplanets," Astrophys. J. 212, 27 (2014).

23. N. Jovanovic et al., "Developing post-coronagraphic, high-resolution spectroscopy for terrestrial planet characterization on ELTs," http://research.iac.es/congreso/AO4ELT5/pages/ proceeding0076.html (2017).

24. T. Kotani et al., "Extremely high-contrast, high spectral resolution spectrometer REACH for the Subaru Telescope," Proc. SPIE 11448, 1144878 (2020).

25. A. Vigan et al., "Bringing high-spectral resolution to VLT/SPHERE with a fiber coupling to VLT/CRIRES+," Proc. SPIE 10702, 1070236 (2018).

26. M. C. Liu et al., "Crossing the Brown Dwarf Desert using adaptive optics: a very close L Dwarf companion to the nearby solar analog HR 7672," Astrophys. J. 571, 519-527 (2002).

27. P. Wizinowich et al., "First light adaptive optics images from the keck II telescope: a new era of high angular resolution imagery," Publ. Astron. Soc. Pac. 112, 315-319 (2000).

28. C. Z. Bond et al., "Adaptive optics with an infrared pyramid wavefront sensor at Keck," J. Astron. Telesc. Instrum. Syst. 6, 039003 (2020).

29. N. Jovanovic et al., "The Keck Planet Imager and Characterizer: demonstrating advanced exoplanet characterization techniques for future extremely large telescopes (Conference Presentation)," Proc. SPIE 11117, 111170 T (2019).

30. J. Pezzato et al., "Status of the Keck Planet Imager and Characterizer phase II development," Proc. SPIE 11117, 111170U (2019).

31. N. Jovanovic et al., "Enhanced high-dispersion coronagraphy with KPIC phase II: design, assembly and status of sub-modules," Proc. SPIE 11447, 114474U (2020).

32. D. Mawet et al., "A fiber injection unit for the Keck Planet Imager and Characterizer," Proc. SPIE 10400, 1040029 (2017).

33. J. Llop-Sayson et al., "Wavefront control experiments with a single mode fiber at the HighContrast Spectroscopy Testbed for Segmented Telescopes (HCST)," Proc. SPIE 11443, 114432Q (2020).

34. C. T. Coker et al., "A multi-object spectrograph using single-mode fibers with a coronagraph: progress towards laboratory results on the high-contrast testbed for segmented telescopes," Proc. SPIE 11117, 111171A (2019).

35. R. K. Gibson et al., "Characterization of the C-RED 2: a high-frame rate near-infrared camera," J. Astron. Telesc. Instrum. Syst. 6(1), 011002 (2019).

36. I. S. McLean et al., "Design and development of NIRSPEC: a near-infrared echelle spectrograph for the Keck II telescope," Proc. SPIE 3354, 566-578 (1998).

37. J. L. Robichaud et al., "Cryogenic performance of the NIRSPEC three-mirror anastigmat," Proc. SPIE 3354, 1068-1076 (1998).

38. E. C. Martin et al., "An overview of the NIRSPEC upgrade for the Keck II telescope," Proc. SPIE 10702, 107020A (2018).

39. "Canada-france-hawaii telescope obervatory manual," https://www.cfht.hawaii.edu/ Instruments/ObservatoryManual/CFHT_ObservatoryManual_\%28Sec_2\%29.html (2003).

40. F. Masci, "Optimum aperture radius for a gaussian profile," https://wise2.ipac.caltech.edu/ staff/fmasci/GaussApRadius.pdf (2008). 
Delorme et al.: Keck Planet Imager and Characterizer: a dedicated single-mode fiber injection unit...

41. R. A. Lopez et al., "Characterization and Performance of the Upgraded NIRSPEC on the W. M. Keck Telescope," Proc. SPIE 11447, 114476B (2020).

42. R. J. Mathar, "Refractive index of humid air in the infrared: model fits," J. Opt. A: Pure Appl. Opt. 9, 470-476 (2007).

43. M. Service et al., "A new distortion solution for NIRC2 on the Keck II telescope," Publ. Astron. Soc. Pac. 128, 095004 (2016).

44. J. J. Wang et al., "Dynamical constraints on the HR 8799 planets with GPI," Astron. J. 156, 192 (2018).

45. J. Wang, M. Kulikauskas, and S. Blunt, "Where is the planet? Predicting positions of directly imaged companions," http://whereistheplanet.com/ (2021).

46. J. R. Crepp et al., "The dynamical mass and three-dimensional orbit of HR7672B: a benchmark Brown Dwarf with high eccentricity," Astrophys. J. 751, 97 (2012).

47. F. Allard, D. Homeier, and B. Freytag, "Models of very-low-mass stars, brown dwarfs and exoplanets," Philos. Trans. R. Soc. Lond. Ser. A 370, 2765-2777 (2012).

48. J.-R. Delorme et al., "The Keck Planet Imager and Characterizer: a dedicated single-mode fiber injection unit for high resolution exoplanet spectroscopy," Proc. SPIE 11447, 114471P (2020).

Biographies of the authors are not available. 